\title{
A simplified meshfree method for shear bands with cohesive surfaces
}

\author{
T.Rabczuk ${ }^{\dagger}$, P.M.A.Areias ${ }^{+}$, T.Belytschko*,\| \\ Department of Mechanical Engineering, Northwestern University, Evanston, IL 60208-311, U.S.A
}

\begin{abstract}
SUMMARY
A simple methodology to model shear bands as strong displacement discontinuities in a meshfree particle method is presented. The shear band is represented as a set of sheared particles. A sheared particle is developed through enrichment by tangential displacement discontinuities. The representation of the shear band as set of cohesive segments provides a simple and versatile model of shear bands. The loss of material stability is used as the criterion for switching from a classical continuum description of the constitutive behavior to a traction-separation law acting on the discontinuity surface. The method is implemented for two and three dimensions. Examples of shear band progression in rate-dependent and rate-independent materials are presented, including the Kalthoff problem, where the transition from brittle fracture to shear banding is studied. Copyright (c) 2000 John Wiley \& Sons, Ltd.
\end{abstract}

KEY WORDS: meshfree methods, shear bands; loss of hyperbolicity; traction-separation laws

\section{INTRODUCTION}

The solution of problems with complex patterns of shear bands in full-scale engineering models still poses a significant challenge since the scale of the local deformation is much smaller than the scale of the complete structure. Furthermore, shear bands are associated with very large strains that are often beyond the capabilities of standard Lagrangian continuum finite element methods. Here, we propose a meshfree method for the evolution of shear bands that can treat complex patterns of shear bands. It will be shown through numerical examples that the proposed method can reproduce the paths of the shear bands and aspects of initiation and arrest as observed experimentally.

Rate independent material models undergoing strain softening manifest bands of infinite strain when hyperbolicity of the linearized Initial Boundary Value Problem (IBVP) is lost. In Bazant and Belytschko [1], a closed form solution was obtained for a wave propagation

*Correspondence to: T. Belytschko, Department of Mechanical Engineering, Northwestern University, 2145 Sheridan Road, Evanston, IL 60208-311, U.S.A. E-mail: t-belytschko@nwu.edu

|| Walter P.Murphy, Professor of Computational Mechanics

$\dagger$ Post-Doctoral Research Fellow, Department of Mechanical Engineering 
problem in a one-dimensional bar with strain softening. It was shown that despite the loss of positivity of the tangent modulus, a solution could be obtained if the region undergoing strain softening was restricted to a set of (Lebesgue) measure zero. This solution is clearly meaningless from a physical point of view, because it implies no dissipation in softening. One way to overcome this pathological behavior is to include a dissipative mechanism in the region in which hyperbolicity has been lost. The results of Bazant and Belytschko [1] also indicated that the region undergoing localized strain can be modelled as a surface through which the displacement field is discontinuous.

Although numerous studies have been pursued to limit the localization by regularization procedures or localization limiters (as in Lasry and Belytschko [2], Needleman and Tvergaard [3]; see Jirasek [4] for a review), regularization does not overcome the basic difficulty that the scale of a shear band is much smaller than the scale of structure. For example, in a $1 \mathrm{~m}$ structure the width of a shear band may be $10^{-5} \mathrm{~m}$, and its structure is quite rich, so 10 to 20 low order elements are needed to adequately resolve its morphology and evolution. Thus a brute force approach to this problem is not viable. An alternative approach employed here is to model shear bands as discontinuities.

The simulation of discontinuities, such as cracks and shear bands, by finite elements or meshfree methods has been pursued by various approaches. One of the most popular classes of methods are the interelement separation models, see Xu and Needleman [5], Camacho and Ortiz [6], Ortiz et al. [7], Zhou and Molinari [8]. These methods were originally developed for cracks and later extended to shear bands, Yang et al. [9]. In these methods, cracks or shear bands are only allowed to develop along existing interelement edges. This provides the method with comparative simplicity, but can result in an overestimate of the dissipated energy when the actual discontinuity paths are not coincident with element edges. Mesh sensitivity has been reported, see Falk et al. [10]; this sensitivity can be mollified by adding randomness to the strength, as in Zhou and Molinari [8].

The extended finite element method is a method that allows the crack or shear band to propagate arbitrarily. Methods for static crack problems in two and three dimensions were developed by Moes et al. [11], Moes and Belytschko [12] and Gravouil et al. [13], respectively. The method was applied to dynamic crack problems in Belytschko et al. [14] and to dislocations in Ventura et al. [15]. A recent paper applies the extended finite element method to discontinuous modelling of shear bands, Samaniego and Belytschko [16]. However, these methods require an explicit representation of the crack or shear band surface, which usually has been provided by level sets. This makes the treatment of phenomena such as branching and fragmentation more difficult, since additional level sets need to be introduced whenever a branch occurs. Another method that does not need a surface representation of the crack or shear band is the embedded discontinuity model (Belytschko et al. [17], Armero and Garikipati [18], Oliver et al. [19]).

Shear bands in meshfree methods have usually been modelled without special treatment other than refinement around the expected paths, see e.g. Li et al. [20, 21], Hao et al. [22]. Cracks in meshfree methods have been treated by Krysl and Belytschko [23] and Organ et al. [24].

In a recent paper, Rabczuk and Belytschko [25] introduced a 'cracking particle' method for modelling arbitrary crack propagation in two dimension; which motivated the proposed method for shear bands. It is implemented in the element free Galerkin-particle (EFG-P) method, Rabczuk and Belytschko [25, 26]. In the proposed method, the shear band is modelled 
by a set of sheared particles. The representation of the shear band by a discrete set of sheared particles dramatically simplifies the method, albeit at some cost in accuracy. The method can be viewed as a local particle partition of unity. The method is of about the same complexity as the interelement separation models of Xu and Needleman [5], less of complex than the interelement method of Ortiz et al. [7] and significantly less complex than the extended finite element method (XFEM).

The transition to sheared particles is governed by a material stability criterion, which in the case of a rate-dependent material is equivalent to loss of hyperbolicity. In this method, a tangential discontinuity in the displacement field is introduced after the loss of material stability. In contrast to crack problems, only displacement jumps parallel to the shear band are allowed.

The paper is organized as follows. First, the approximation of the displacement field is described. The use of the local material stability of the momentum equations is used as a shear band initiation indicator. Then, the formulation of a dissipative interface is described in section 3, along with a B-bar approach with selective reduced integration (to remove volumetric locking) of the EFG-P method in section 4. Results for a variety of problems compared to experimental results are given in section 5 .

\section{DISPLACEMENT FIELD AND WEAK FORM}

The shear band is modelled by a set of discrete shear bands as shown in figure 1, i.e. by a set of sheared particles. A standard meshfree methodology based on moving least square approximations (Belytschko et al. [27], Rabczuk et al. [28]) with a Lagrangian kernel is used; the latter is essential for avoiding artificial discretization instabilities known as tensile instabilities which often result in spurious, numerically induced fracture in SPH methods.

The velocity field is additively partitioned into a continuous part and a discontinuous part by

$$
\dot{\mathbf{u}}(\mathbf{X}, t)=\dot{\mathbf{u}}^{\text {cont }}(\mathbf{X}, t)+\dot{\mathbf{u}}^{d i s c}(\mathbf{X}, t)
$$

where $\mathbf{u} \in \Re^{n_{S D}}$ is the displacement, $\mathbf{X} \in \Re^{n_{S D}}$ are the material coordinates, $t$ is the time and $n_{S D}$ the number of space dimensions and the superimposed dots denote time derivatives.

Let the set of sheared particles be denoted by $\mathcal{N}_{s}$ and the total set of particles by $\mathcal{N}$. The sheared particles are determined by a stability criterion described later. For each sheared particle we assume that the normal to the shearing plane $\mathbf{n}$ is provided by a material stability analysis or some other material failure criterion. This enables us to construct a set of tangent vectors $\mathbf{e}_{\alpha T}, \alpha=1$ to $n_{S D}-1$, such that $\mathbf{e}_{\alpha T} \cdot \mathbf{n}=0$. The continuous and discontinuous displacement fields are then given by

$$
\begin{aligned}
\dot{\mathbf{u}}^{\text {cont }}(\mathbf{X}, t) & =\sum_{I \in \mathcal{N}} \Phi_{I}(\mathbf{X}) \dot{\mathbf{u}}_{I}(t) \\
\dot{\mathbf{u}}^{d i s c}(\mathbf{X}, t) & =\sum_{I \in \mathcal{N}_{s}} \sum_{\alpha=1}^{n_{S D}-1} \Phi_{I}(\mathbf{X}) S\left(f_{I}(\mathbf{x})\right) \dot{q}_{\alpha I}(t) \mathbf{e}_{\alpha T}
\end{aligned}
$$

where $\Phi_{I}(\mathbf{X})$ are the moving least squares (MLS) shape functions, Belytschko et al. [29], $\mathbf{u}_{I}(t)$ are the nodal displacements (only approximately; MLS shape functions are not interpolants), 


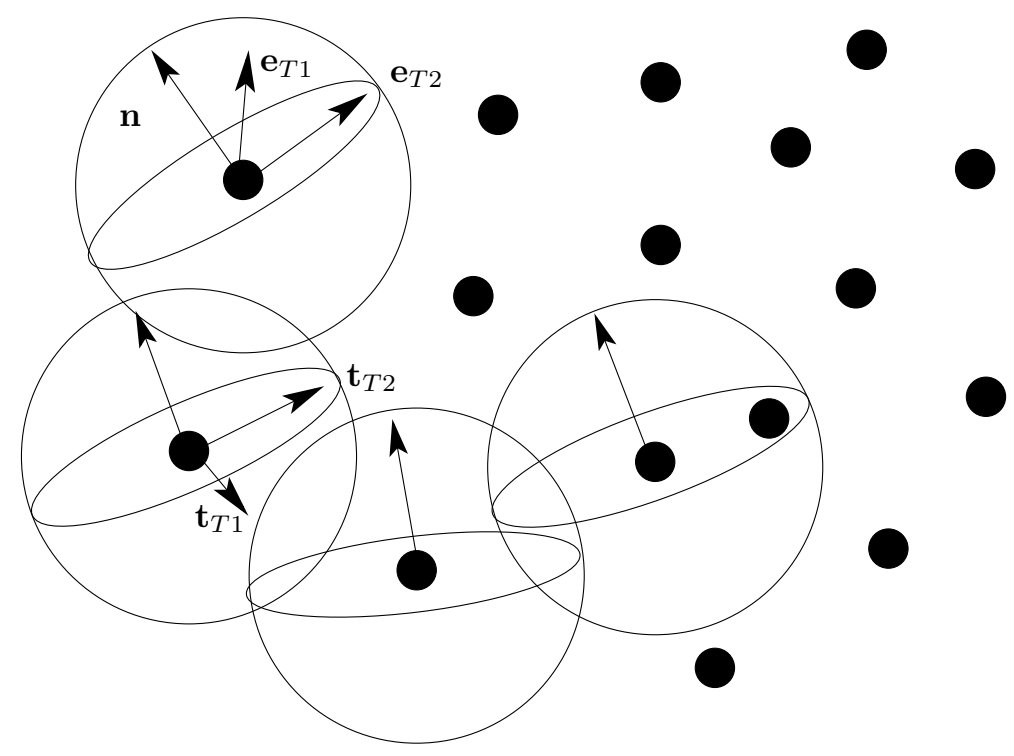

Figure 1. A set of sheared particles; the radius of the domain of influence is reduced compared to the actual size for clarity.

$S\left(f_{I}(\mathbf{x})\right)$ is the sign function defined by

$$
S(\xi)=\left\{\begin{array}{cc}
1 & \text { if } \xi>0 \\
-1 & \text { if } \xi<0
\end{array}\right.
$$

and $f_{I}(\mathbf{x})$ is given by

$$
f_{I}(\mathbf{x})=\mathbf{n} \cdot\left(\mathbf{x}-\mathbf{x}_{I}\right)
$$

where $\mathbf{x}=\mathbf{X}+\mathbf{u}$ and $\mathbf{x}_{I}$ is the current position of the node $I$. The nomenclature is illustrated in figure 1 .

The role of the sheared particles is to mimic a displacement field with a jump in the tangential displacements. To represent a linear tangential jump, there must be a sufficient number of sheared particles so that the linear completeness of the shape function is inherited by the jump field. In this work, in contrast to Rabczuk and Belytschko [25], a linear basis was chosen for the EFG-P shape functions employed for the discontinuous field.

Consider a solid $\Omega$ with material points $\mathbf{X}$, see figure 2, Its boundary $\Gamma$ is partitioned into two subsets, $\Gamma^{t}$ and $\Gamma^{u}$, upon which tractions and displacements are applied, the corresponding entities in the reference configuration are denoted by $\Omega_{0}, \Gamma_{0}^{t}$ and $\Gamma_{0}^{u}$. The internal surface of discontinuity $\Gamma^{s}$ is approximated by local discontinuities $\Gamma_{I}^{s}$; in the following $\Gamma^{s}=\bigcup_{I} \Gamma_{I}^{s}$.

We will consider for a given time $t$ the space $S_{t}$ of suitably smooth functions, i.e. $C^{0}$ functions. Let us also define the corresponding space of trial and test functions as

$$
\begin{aligned}
S_{t} & :=\left\{\mathbf{u}_{t} \mid \mathbf{u}_{t} \in C^{0} \text { except on } \Gamma^{s}, \mathbf{u}_{t}=\overline{\mathbf{u}} \text { on } \Gamma^{u}\right\} \\
V_{t} & :=\left\{\delta \mathbf{u} \mid \delta \mathbf{u} \in S_{t}, \overline{\mathbf{u}}=0 \text { on } \Gamma^{u}\right\}
\end{aligned}
$$




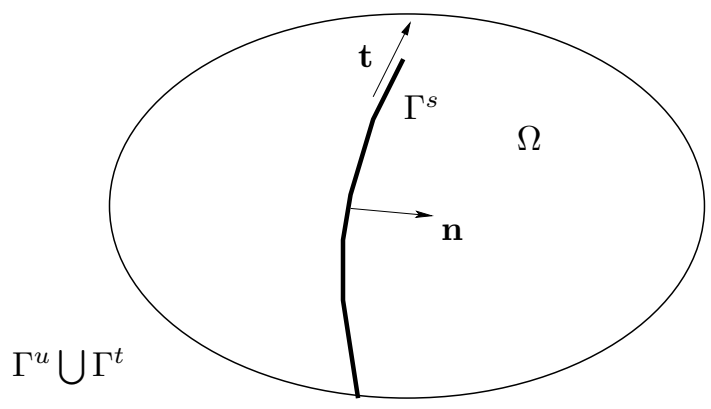

a)

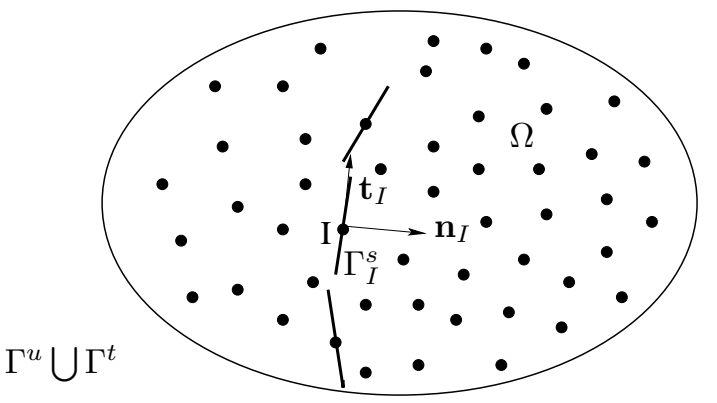

b)

Figure 2. Body with a shear band $\Gamma^{s}$ and its representation of $\Gamma^{s}$ by the cracked particle method

Now, we state the following weak form of an IBVP for a solid with an internal dissipative interface $\Gamma^{s}$ :

GIVEN the initial displacement, $\mathbf{u}_{0}$, and the initial stress, $\boldsymbol{\sigma}_{0}$,

FIND $\mathbf{u}_{t} \in S_{t}$ for every time $t$ in the time interval of analysis

SUCH THAT, for all $\delta \mathbf{u} \in V_{t}$,

$$
\left.\int_{\Omega} \rho \delta \mathbf{u} \cdot \ddot{\mathbf{u}} d \Omega=\int_{\Omega_{0} \backslash \Gamma^{s}} \nabla_{0} \otimes \delta \mathbf{u}^{S}: \boldsymbol{\sigma} d \Omega-\int_{\Omega} \delta \mathbf{u} \cdot \mathbf{b} d \Omega-\int_{\Gamma^{t}} \delta \mathbf{u} \cdot \mathbf{t} d \Gamma+\int_{\Gamma^{s}} \delta \llbracket \mathbf{u} \rrbracket \cdot \mathbf{t}^{s}\right\} d \Gamma
$$

in the time interval of the analysis where $\rho$ is the mass density, the superimposed $S$ denotes the symmetric part of the tensor and $\boldsymbol{\sigma}$ is the Cauchy stress.

\section{SHEAR BAND MODEL}

\subsection{Shear band initiation}

Once the material loses stability, the standard governing equations are no longer applicable because they do not model the physical energy dissipation process. Several remedies to reintroduce the dissipated energy in the model have been proposed. One of them is to include a surface dissipative mechanism once the material looses stability, see Belytschko et al. [14], Wang et al. [30].

Within the context of nonlinear fracture mechanics, this dissipative mechanism has been introduced by means of the so-called cohesive surfaces. We adopt a similar methodology here for modelling shear bands.

Shear bands have been shown to be a consequence of softening by Clifton et al. [31] and Wright and Walter [32]. In a rate-dependent material, the PDE does not change type and only undergoes a material instability, whereas in a rate independent material, it loses hyperbolicity. A classical definition of material stability is the Legendre-Hadamard condition, which establishes that for any nonzero vectors $\mathbf{n}$ and $\mathbf{h}$ the following point-wise inequality 
must hold for material stability:

$$
(\mathbf{n} \otimes \mathbf{h}): \mathbf{A}:(\mathbf{n} \otimes \mathbf{h})>0 \quad \forall \mathbf{n} \text { and } \forall \mathbf{h}
$$

where $\mathbf{A}$ is related to the constitutive tangent operator for the Truesdell rate, see Belytschko et al. [33]:

$$
\mathbf{A}=\mathbf{C}+\boldsymbol{\sigma} \otimes \mathbf{I}
$$

The vector $\mathbf{n}$ defines the direction of propagation and $\mathbf{h}$ is the polarization of the wave. This condition ensures that the velocity of propagating waves in a solid remains real. Equality in expression (7) is the necessary condition for stationary waves. The Legendre-Hadamard condition is sometimes called the strong ellipticity condition, since it ensures the ellipticity of the equilibrium equation for rate-independent materials. In the dynamical case, the satisfaction of the Legendre-Hadamard condition implies the hyperbolicity of the IBVP. The reader is referred to Silhavy [34] for details about the concepts mentioned above.

Based on (7), let us define for a given material point of a solid at a given time the acoustic tensor

$$
\mathbf{Q}=\mathbf{n} \cdot \mathbf{A} \cdot \mathbf{n}
$$

Equation (7) is equivalent to the condition that the minimum eigenvalue of $\mathbf{Q}$ is strictly positive, so we say a material point is stable whenever the minimum eigenvalue of $\mathbf{Q}$ is strictly positive and unstable otherwise.

There are certain difficulties in finding the direction of the shear band with this approach. First of all, since $\mathbf{n}=(\cos \alpha \cos \beta, \cos \alpha \sin \beta, \sin \alpha)$ depends on two angles in three dimensions, the computation of the minimum eigenvalues of $\mathbf{Q}$ as a function of the angles becomes computationally expensive. We have checked the minimum eigenvalue of $\mathbf{Q}$ for every 4 degrees in both angular variables and used parallelization to keep the computational cost low. A typical picture of the minimum eigenvalues as a function of the two angles at one material point is shown in figure 3. As can be seen, the eigenvalue landscape is very complex with many local minima and exactly four absolute minima.

The second difficulty is that four angles are obtained (only two of them are relevant, since the other two are in the same direction but of opposite sense). We choose the direction of the maximum displacement gradient by maximizing

$$
g=\underbrace{\max }_{l}\left(\mathbf{n}_{l}^{T} \cdot\left(\nabla \mathbf{u} \cdot \mathbf{e}_{T \alpha I}\right)\right)
$$

where the normals $\mathbf{n}_{l}$ correspond to minima of $\mathbf{Q}$, eq. (9). The normal in the initial configuration is obtained by Nanson's law:

$$
\mathbf{n}_{0}=J^{-1} \mathbf{n}\left(t_{c r}\right) \cdot \mathbf{F}\left(\mathbf{X}_{I}, t_{c r}\right)
$$

where $t_{c r}$ is the time of loss-of-stability, and the normal at any time $t$ is computed by the inverse of eq. (11).

Difficulties sometimes occur at shear band intersections. In this case, the shear band orientation is rotated into a new orientation to allow the new band to propagate. This is accomplished by switching the angles of the sheared particles to the angle of the crossing or reflecting shear band. 


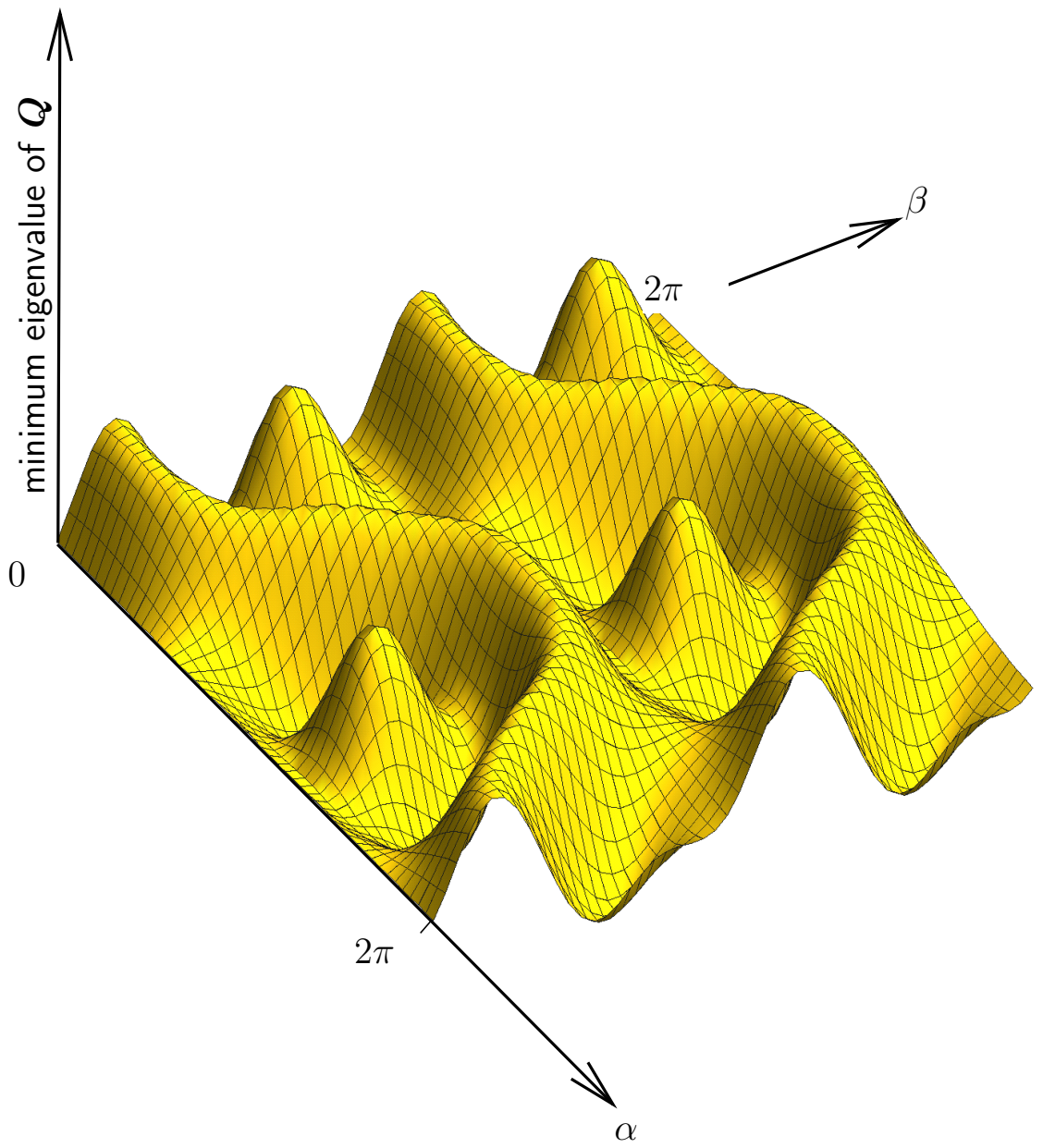

Figure 3. Minimum eigenvalue of the acoustic tensor as a function of the two angles for one material point

\subsection{Interface model}

The interface law is a cohesive model which relates the tangential components of the interface force $\mathbf{t}_{T}$ to the jump; i.e. the magnitude of the discontinuity, in the interface tangent displacement or velocity, $\left[\left[\mathbf{u}_{T}\right]\right]$ or $\left[\left[\dot{\mathbf{u}}_{T}\right]\right]$, respectively. These are called the slip and slip rate, respectively. The magnitude of the slip is given by

$$
\left|[[u]]_{T}\right|=\left([[u]]_{1 T}^{2}+[[u]]_{2 T}^{2}\right)^{1 / 2}
$$

whereas the magnitude of the slip rate is given by

$$
\left|[[\dot{u}]]_{T}\right|=\left([[\dot{u}]]_{1 T}^{2}+[[\dot{u}]]_{2 T}^{2}\right)^{1 / 2}
$$


Interface behavior can reflect a large variety of physical phenomena. In rock and granular materials, it is probably largely frictional in character. On the other hand, in metals, the interface law is an idealization of a complex process that involves coupled heat conduction and elastic-plastic behavior that often is highly temperature dependent. It is not clear that this complexity can be embodied in an interface law. However, we have found that the patterns and onset of shear bands can be captured by relatively simple laws.

The form of the law we have used is illustrated in figure 4. As can be seen, the value of the cohesive force at the nucleation of the shear discontinuity corresponds to the shear stress across the plane normal to $\mathbf{n}$ at that time of nucleation $\sigma_{\text {Tmax }}$. Subsequently, it decreases linearly to zero, although other decay functions can easily be implemented.

The dissipation by the shear band is $D_{s}$ as shown in figure 4. Several authors have made estimates of this dissipated energy. For example, Grady [35] and Minnaar and Zhou [36] give the dissipation energy as

$$
D_{s}=\frac{\varrho c_{v}}{\alpha}\left(\frac{9 \varrho^{3} c_{v}^{2} \chi^{3}}{\sigma_{y}^{3} \alpha^{2} \dot{\gamma}}\right)^{1 / 4}
$$

where $\varrho$ is the density, $\chi$ is the thermal diffusion coefficient, $\sigma_{y}$ is the flow stress at strain $\dot{\gamma}_{y}$, $\alpha$ is a thermal softening coefficient and $c_{v}$ is the specific heat.

The conditions for loading/unloading/reloading are

$$
\begin{array}{r}
\left|[[u]]_{T}\right|^{n+1}>\left|[[u]]_{T}\right|^{n}>\left|[[u]]_{T}\right|^{\max } \text { loading } \\
\left|[[u]]_{T}\right|^{n+1}>\left|[[u]]_{T}\right|^{n} \leq\left|[[u]]_{T}\right|^{\text {max }} \text { reloading } \\
\left|[[u]]_{T}\right|^{n+1} \leq\left|[[u]]_{T}\right|^{n} \text { unloading }
\end{array}
$$

where the superscript denotes the time step. In unloading, an elastic slope based on the shear band width is used.

The primary curve for the linear decay law shown in figure 4 a is given by

$$
t_{T}=\sigma_{T \max }-\frac{\sigma_{T \max }}{\delta_{T \max }}[[u]]_{T}
$$

The rates of the tangential tractions are obtained by an adaptation of strain space plasticity, see for example Nemat-Nasser [37]. The strain-based yield function in the plane of the shear band is given by

$$
[[u]]_{1 T}^{2}+[[u]]_{2 T}^{2}-j^{2}=0
$$

where $j$ measures the total slip. The above is related to $J_{2}$ plasticity: in the shear band, the slip components are related to the shear strain components, and a strain-based yield function would take the above form. We adopt a rigid plastic formalism for the loading law, so

$$
\dot{t}_{\alpha T}=c[[\dot{u}]]_{\alpha T}
$$

where

$$
c=\left\{\begin{array}{c}
\text { slope of cohesive law in loading } \\
\text { slope of unloading/reloading curve }
\end{array}\right.
$$

For the linear cohesive law at loading conditions

$$
c=-\frac{\sigma_{T \max }}{\delta_{T \max }}
$$

In most cases, we used a linear cohesive curve as in figure $4 \mathrm{a}$ and for metals we set $\delta_{T \text { max }}$ by equating the energy (14) to the area under the cohesive curve. 


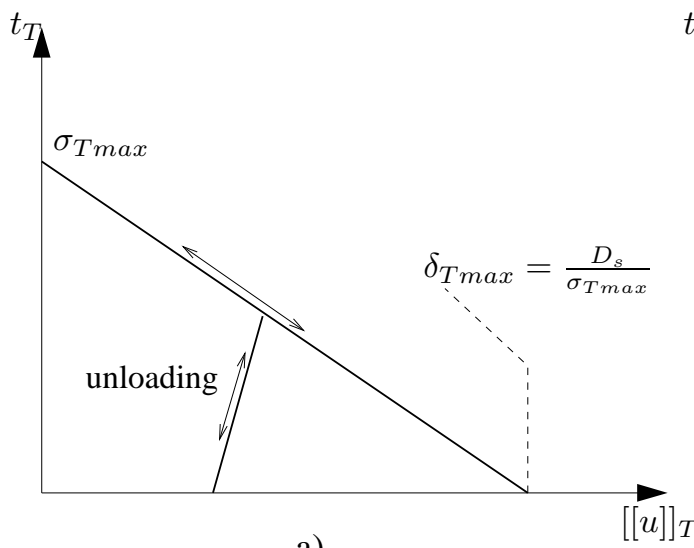

a)

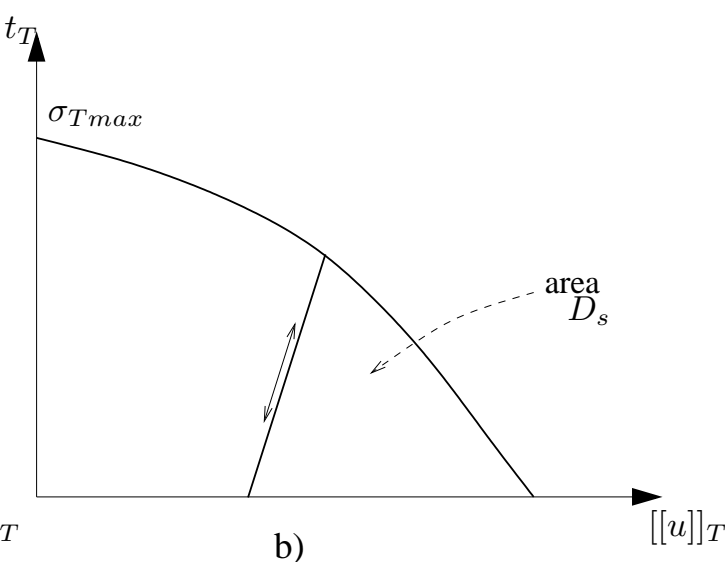

b)

Figure 4. Cohesive laws used in computations: a) linear; b) bilinear.

\section{DISCRETIZATION}

\subsection{Approximation of the pressure field}

Locking in meshfree methods has been considered by Dolbow and Belytschko [38] and Chen et al. [39]. To avoid locking, we will use a variant of the B-bar formulation [40]. The deformation gradient is decomposed into a deviatoric and volumetric part, see Flory [41]:

$$
\mathbf{F}=\mathbf{F}^{\text {dev }} \mathbf{F}^{\text {vol }}
$$

The approximations for $\mathbf{F}$ and $\overline{\mathbf{F}}$ are given by

$$
\begin{aligned}
\mathbf{F} & =\sum_{I \in \mathcal{N}} \nabla \Phi_{I}(\mathbf{X}) \mathbf{u}_{I}(t)+\sum_{I \in \mathcal{N}_{c}} \nabla \Phi_{I}(\mathbf{X}) S\left(f_{I}(\mathbf{X})\right) q_{\alpha I}(t) \mathbf{e}_{\alpha T}(\mathbf{X})+\mathbf{I} \\
\overline{\mathbf{F}} & =\sum_{I \in \mathcal{N}} \nabla \bar{\Phi}_{I}(\mathbf{X}) \mathbf{u}_{I}(t)+\sum_{I \in \mathcal{N}_{c}} \nabla \bar{\Phi}_{I}(\mathbf{X}) S\left(f_{I}(\mathbf{X})\right) q_{\alpha I}(t) \mathbf{e}_{\alpha T}(\mathbf{X})+\mathbf{I}
\end{aligned}
$$

where $\Phi_{I}(\mathbf{X})$ are shape functions based on a bilinear basis and the shape functions $\bar{\Phi}_{I}(\mathbf{X})$ use a constant basis. The facts that $\nabla S\left(f_{I}(\mathbf{X})\right)$ and $\nabla e_{\alpha T}$ vanish are already incorporated into eq. (22). The former term vanishes since the shear band is considered as an open set and the latter since the discontinuity is piecewise constant. The volumetric part of the deformation gradient can then be computed in terms of the constant-basis deformation gradient $\overline{\mathbf{F}}$ by

$$
\overline{\mathbf{F}}^{\text {vol }}=\frac{1}{3} \operatorname{tr}(\overline{\mathbf{F}}) \mathbf{I}
$$

The pressure $\bar{p}$ is computed using the following constitutive law

$$
\bar{p}=K(\theta-1)
$$

with

$$
\theta=\operatorname{det}(\overline{\mathbf{F}})
$$


The deviatoric deformation gradient is computed by

$$
\mathbf{F}^{\text {dev }}=\mathbf{F}\left(\mathbf{F}^{v o l}\right)^{-1}
$$

Since meshfree shape functions with a bilinear basis are used for $\Phi_{I}(\mathbf{X})$ and shape functions with constant basis for $\bar{\Phi}_{I}(\mathbf{X})$, this can lead to instabilities. They can be eliminated by a stabilization procedure such as GLS or SUPG, Brooks and Hughes [42], Hughes et al. [43]. The instabilities can be also prevented by using higher order shape functions. However, we won't address the issue of pressure instabilities within this paper.

\subsection{B-Bar formulation}

Substituting the approximations of the test and trial functions developed in section 2 and invoking the arbitrariness of $\delta \mathbf{u}_{I}$ and $\delta \mathbf{q}_{\alpha I}$ into eq. (6) we obtain

$$
\begin{gathered}
\sum_{I \in \mathcal{N}} \delta \mathbf{u}_{I} \cdot\left(\mathbf{f}_{I}^{i n t}+\mathbf{M}_{I K}^{u u} \cdot \ddot{\mathbf{u}}_{K}+\mathbf{M}_{I K}^{u q_{1}} \ddot{q}_{1 K}+\mathbf{M}_{I K}^{u q_{2}} \ddot{q}_{2 K}-\mathbf{f}_{I}^{e x t}\right)+ \\
\sum_{I \in \mathcal{N}_{c}} \delta q_{1 I}\left(Q_{1 I}^{i n t}+\mathbf{M}_{I K}^{u q_{1}} \cdot \ddot{\mathbf{u}}_{K}+\mathbf{M}_{I K}^{q_{1} q_{1}} \ddot{q}_{1 K}+\mathbf{M}_{I K}^{q_{1} q_{2}} \ddot{q}_{2 K}-Q_{1 I}^{e x t}\right)+ \\
\sum_{I \in \mathcal{N}_{c}} \delta q_{2 I}\left(Q_{2 I}^{i n t}+\mathbf{M}_{I K}^{u q_{2}} \cdot \ddot{\mathbf{u}}_{K}+\mathbf{M}_{I K}^{q_{1} q_{2}} \ddot{q}_{1 K}+\mathbf{M}_{I K}^{q_{2} q_{2}} \ddot{q}_{2 K}-Q_{2 I}^{e x t}\right)=0
\end{gathered}
$$

where

$$
\begin{gathered}
\mathbf{f}_{I}^{\text {int }}=\int_{\Omega \backslash \Gamma^{s}} \nabla \Phi_{I}(\mathbf{X}) \cdot \operatorname{dev}(\boldsymbol{\sigma}(\mathbf{X})) d \Omega+\int_{\Omega \backslash \Gamma^{s}} \nabla \bar{\Phi}_{I}(\mathbf{X}) \cdot \mathbf{I} \bar{p}(\mathbf{X}) d \Omega \\
\mathbf{f}_{I}^{e x t}=\int_{\Omega \backslash \Gamma^{s}} \varrho \mathbf{b} \Phi_{I}(\mathbf{X}) d \Omega^{+} \int_{\Gamma^{t}} \overline{\mathbf{t}} \Phi_{I}(\mathbf{X}) d \Gamma \\
Q_{\alpha I}^{i n t}=\int_{\Omega \backslash \Gamma^{s}} S\left(f_{I}(\mathbf{X})\right)\left(\nabla \Phi_{I}(\mathbf{X}) \otimes \mathbf{e}_{\alpha T}\right)^{S}: \operatorname{dev}(\boldsymbol{\sigma}(\mathbf{X})) d \Omega+\underbrace{\int_{\Omega \backslash \Gamma^{s}} S\left(f_{I}(\mathbf{X})\right) \Phi_{I}(\mathbf{X})\left(\nabla \otimes \mathbf{e}_{\alpha T}\right)^{S}: \operatorname{dev}(\boldsymbol{\sigma}(\mathbf{X})) d \Omega}_{=0} \\
+\int_{\Omega \backslash \Gamma^{s}} S\left(f_{I}(\mathbf{X})\right)\left(\nabla \bar{\Phi}_{I}(\mathbf{X}) \otimes \mathbf{e}_{\alpha T}\right)^{S}: \mathbf{I} \bar{p}(\mathbf{X}) d \Omega+\underbrace{\int_{\Omega \backslash \Gamma^{s}} S\left(f_{I}(\mathbf{X})\right) \bar{\Phi}_{I}(\mathbf{X})\left(\nabla \otimes \mathbf{e}_{\alpha T}\right)^{S}: \mathbf{I} \bar{p}(\mathbf{X}) d \Omega}_{=0}(30) \\
Q_{\alpha I}^{e x t}=\int_{\Omega \backslash \Gamma^{s}} \varrho\left(\mathbf{b} \cdot \mathbf{e}_{\alpha T}\right) S\left(f_{I}(\mathbf{X})\right) \Phi_{I}(\mathbf{X}) d \Omega^{+} \int_{\Gamma^{t}}\left(\overline{\mathbf{t}} \cdot \mathbf{e}_{\alpha T}\right)
\end{gathered}
$$

where the spatial derivatives with respect to $\mathbf{e}_{\alpha T}, \alpha=1,2$ vanish as indicated above since the discontinuity is piecewise constant. Each of the parenthesis in eq. (27) gives a discrete equation of motion.

The mass matrix is given by

$$
\mathbf{M}_{I J}=\left[\begin{array}{ccc}
\mathbf{m}_{I J}^{u u} & \mathbf{m}_{I J}^{u q_{1}} & \mathbf{m}_{I J}^{u q_{2}} \\
\mathbf{m}_{I J}^{u q_{1}} & \mathbf{m}_{I J}^{q_{1} q_{1}} & \mathbf{m}_{I J}^{q_{1} q_{2}} \\
\mathbf{m}_{I J}^{u q_{2}} & \mathbf{m}_{I J}^{q_{1} q_{2}} & \mathbf{m}_{I J}^{q_{2} q_{2}}
\end{array}\right]
$$


with

$$
\begin{aligned}
\mathbf{m}_{I J}^{u u} & =\int_{\Omega \backslash \Gamma^{s}} \varrho_{0} \Phi_{I}(\mathbf{X}) \hat{\Phi}_{J}(\mathbf{X}) \mathbf{I} d \Omega \\
\mathbf{m}_{I J}^{u q_{\alpha}} & =\int_{\Omega \backslash \Gamma^{s}} \varrho_{0} \Phi_{I}(\mathbf{X}) \Phi_{J}(\mathbf{X}) S\left(f_{J}(\mathbf{X})\right) \mathbf{e}_{\alpha T} d \Omega \\
\mathbf{m}_{I J}^{q_{\alpha} q_{\alpha}} & =\int_{\Omega \backslash \Gamma^{s}} \varrho_{0} \Phi_{I}(\mathbf{X}) S\left(f_{I}(\mathbf{X})\right) \Phi_{J}(\mathbf{X}) S\left(f_{J}(\mathbf{X})\right) d \Omega
\end{aligned}
$$

A selective reduced integration is chosen. For the part of the nodal internal forces that depends on the pressure, the second term in eqs. (28) and (30), we employed a nodal integration:

$$
\int_{\Omega_{0}} \Phi_{I}(\mathbf{X}) g(\mathbf{X}) d \Omega_{0}=\sum_{J \in \mathcal{N}_{I}} \Phi_{I}\left(\mathbf{X}_{J}\right) g\left(\mathbf{X}_{J}\right) V_{J}^{0}
$$

where $V_{J}^{0}$ is the Voronoi volume of node $J$ and $\mathcal{N}_{I}$ is the set of nodes in the support of $\Phi_{I}(\mathbf{X})$. Stress point integration is used for the deviatoric terms. A stress point is arranged in the middle of each cell (in 2D) or cuboid (in 3D) spanned by four and eight particles, respectively; see Rabczuk and Belytschko [25], Rabczuk et al. [28] for our construction of stress points. One point quadrature was used for the surface integrals $\Gamma_{I}^{s}$

\section{EXAMPLES}

\subsection{Plate under tension}

Consider a plate under uniaxial tension with notches as shown in figure 5 , We use $J_{2}$ plasticity and a bilinear cohesive model. Young's modulus for this problem is $E=210,000 \mathrm{MPa}$, Poisson's ratio $\nu=0.3$, yield strength $f_{y}=200 \mathrm{MPa}$ and softening modulus $H=$ $-10,000 M P a$. The load is applied as a displacement boundary condition on a layer of finite elements.

We tested different refinements and will present the results for 1,350,000 particles and 180,000 particles though good results were obtained also with coarser models. First, we will consider the plate where the notch is $5 \mathrm{~mm}$ above the bottom. A constant velocity of $5 \mathrm{~m} / \mathrm{s}$ was applied at the top surface. The shear band at different time steps is shown in figures $6 \mathrm{a}, \mathrm{b}, \mathrm{c}, \mathrm{d}$ and 6 e, f,g,h for the 180,000 and 1,350,000 particle discretizations, respectively. It can be seen that the pattern is almost completely independent of the degree of refinement.

At first, the shear band propagates downward at a 45 degree angle. As can be seen from figure 7, the shear band velocity is quite small at this time due to the short distance to the lower boundary which may reduce the driving force for the shear band. At approximately 0.25 $m s$, the shear band is reflected at the lower boundary and starts to propagate upward at 45 degrees. At approximately $0.5 \mathrm{~ms}$ the shear band reaches the right vertical boundary; as can be seen from figure 7, the shear band tip then accelerates. A large rise in the shear band velocity followed by a sudden drop is observed. Evidently the shear band tip is accelerated as it nears the right boundary.

The shear band speed is obtained by recording the coordinates of the sheared particles and computing the distance between the shear band tip (as indicated by the farthest enriched 


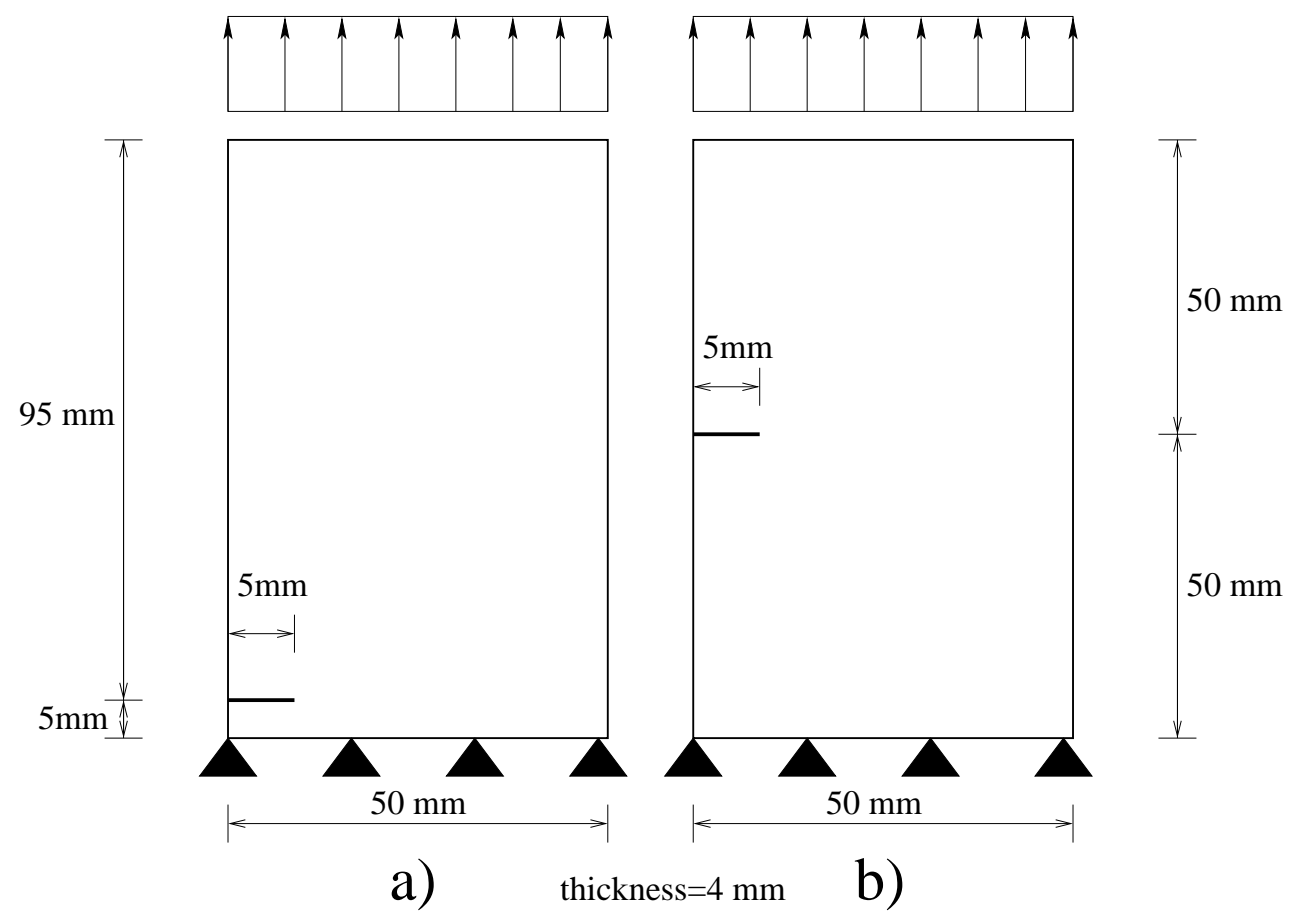

Figure 5. Plate under tension with notches at different locations

particle) every $5 \mu \mathrm{s}$. Hence, these are only approximate values. The shear band velocity is below the Rayleigh wave speed.

The effective plastic strain at different time steps for the 180,000 particle discretization is shown in figure 8; the results for the finer discretization look similar. Moderate plastic strains appear to occur at a substantial distance from the shear band.

Next, we will consider the plate with an initial crack in the middle of the specimen, see figure 5b. We prescribed the same velocity at the top. We will present the results for the 180,000 particle discretization. The shear band at different time steps is shown in figure 9. The shear band propagates downward from the notch at 45 degrees and reaches the right side of the specimen at about $0.06 \mathrm{~ms}$. A second shear band then starts to propagate upward from the notch a short time before the other shear band reaches the right wall. Finally, a third shear band initiates at the right wall of the specimen and starts to propagate towards the second shear band; it can just barely be seen in figure $9 \mathrm{c}$. At the end of the computation, $1 \mathrm{~ms}$, the specimen is sheared almost into three pieces.

\subsection{The Kalthoff experiment}

Kalthoff and Winkler [44] and Kalthoff [45] performed a series of experiments where a steel plate is subjected to impact loading with different impact velocities as shown in figure 10. They discovered that the failure mode depends on the impact velocity. Up to a certain velocity of 


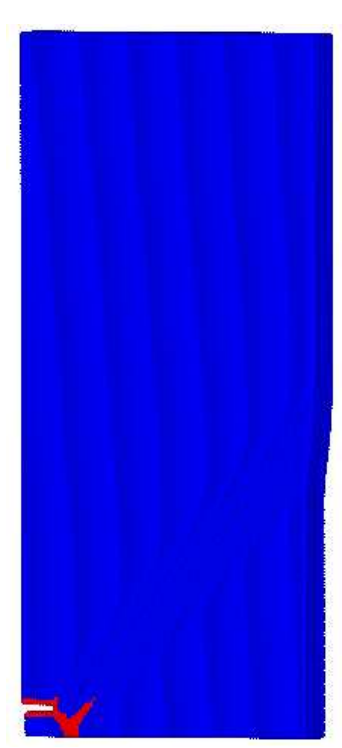

a) $0.35 \mathrm{~ms}$

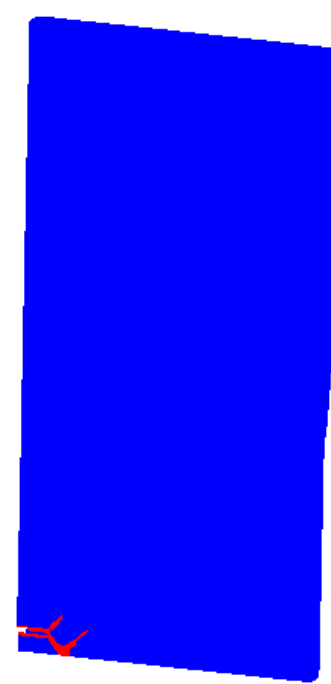

e) $0.35 \mathrm{~ms}$

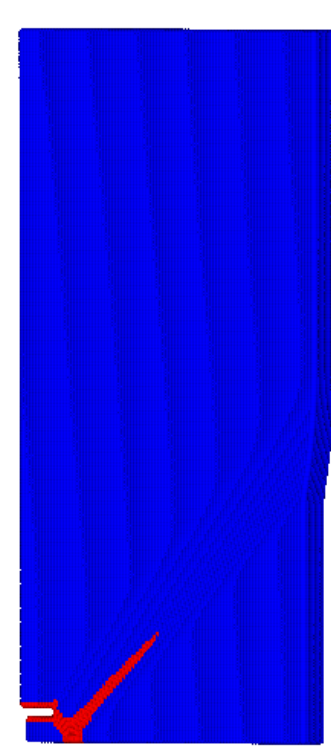

b) $0.42 \mathrm{~ms}$

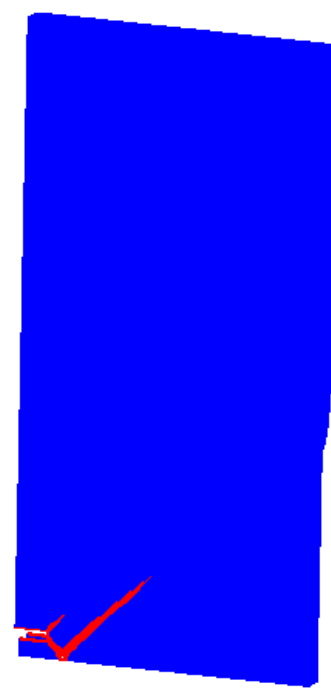

f) $0.42 \mathrm{~ms}$

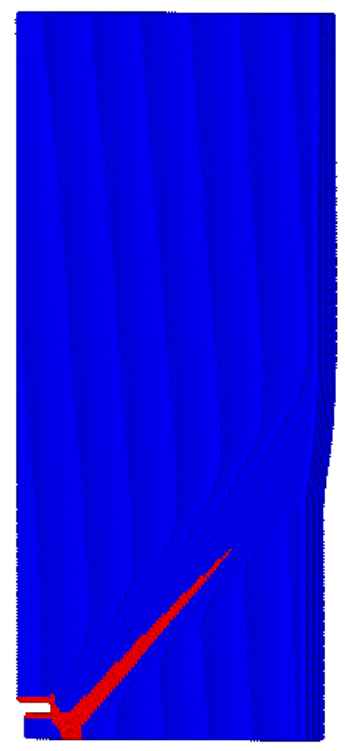

c) $0.46 \mathrm{~ms}$

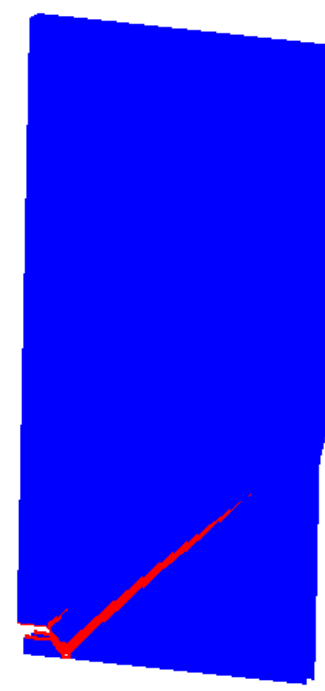

g) $0.46 \mathrm{~ms}$

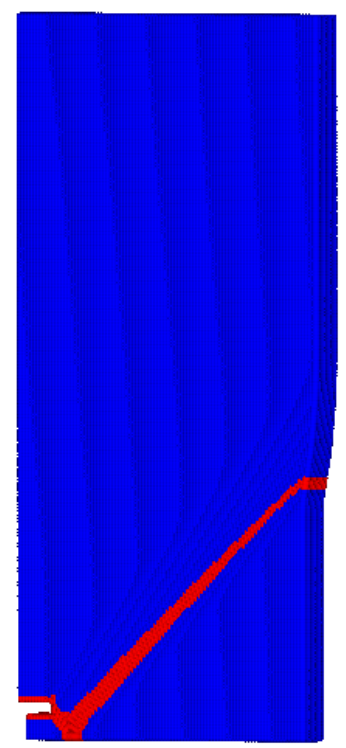

d) $0.5 \mathrm{~ms}$

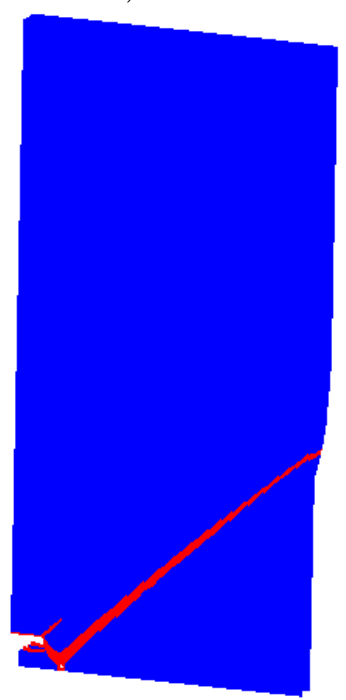

h) $0.5 \mathrm{~ms}$

Figure 6. Deformation of a specimen subjected to tensile loading at different time steps for a) to d) 180,000 particles and e) to h) 1,350,000 particles 


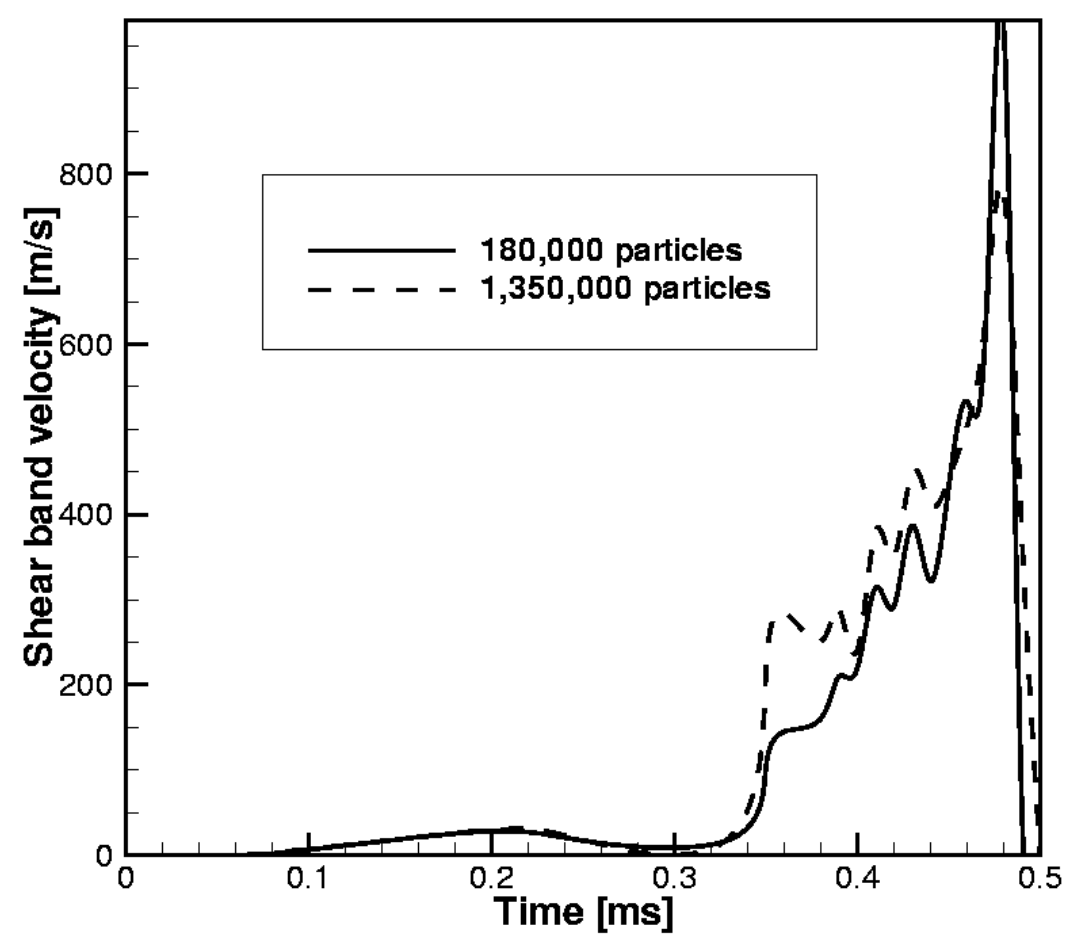

Figure 7. Shear band tip velocity for the plate with the notch close to the support

the impactor $v_{c}$, the steel plate undergoes brittle fracture: a crack develops at a 70 degree angle to the horizontal axis. When exceeding $v_{c}$, they found a shear band developed from the notch. We will focus on experiments done with steel and sharp notches with small radius, so that we expect failure mode transition with $v_{c}=30 \mathrm{~m} / \mathrm{s}$.

After Kalthoff and Winkler [44] published their results, a series of similar impact experiments were carried out that in general confirm their observations. However, in similar experiments performed by Zhou et al. [46], no failure was observed at low impact speed. For high impact speeds, a shear band starts to propagate nearly parallel to the notch, is arrested later and becomes a crack. Experiments on polycarbonite by Ravi-Chandar et al. [47] revealed a failure mode transition from ductile to brittle at an impact speed of $29 \mathrm{~m} / \mathrm{s}$ and another transition from brittle to ductile at impact speeds higher than $55 \mathrm{~m} / \mathrm{s}$.

Numerical studies of the Kalthoff and related experiments include Needleman and Tvergaard [3], Li et al. [20], Batra and Gummalla [48], Zhou et al. [49]. Mostly two-dimensional computations under the assumption of plane strain conditions were performed. The impact was usually modelled by a velocity boundary condition. Three-dimensional simulations have been made, e.g. by Batra and Ravisankar [50].

In our computations we used the Johnson-Cook model [51] with $J_{2}$ plasticity because strain rate and temperature effects play an important role. The effective yield stress of the Johnson- 


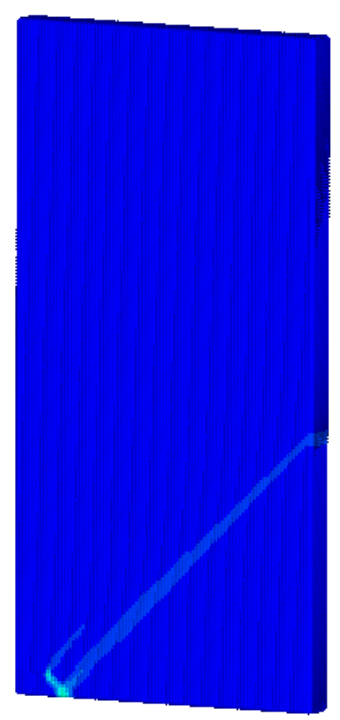

a) $0.07 \mathrm{~ms}$

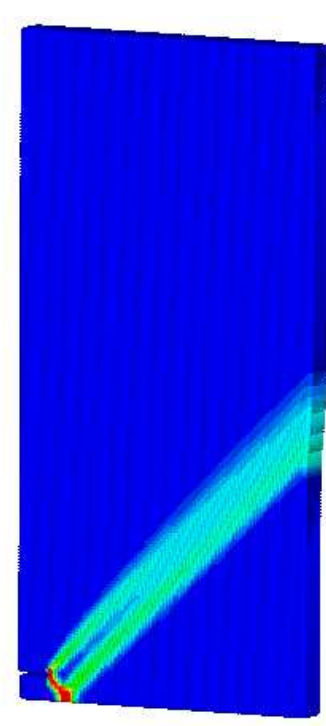

b) $0.25 \mathrm{~ms}$

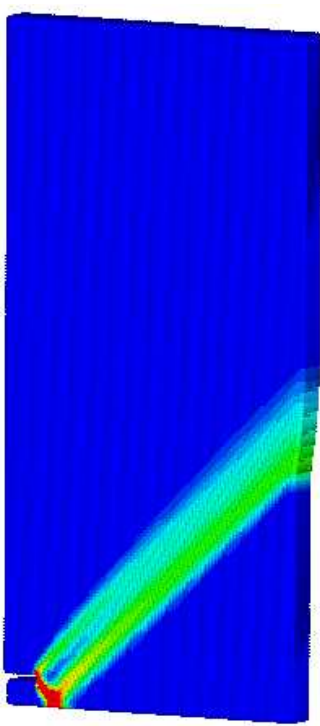

c) $0.35 \mathrm{~ms}$

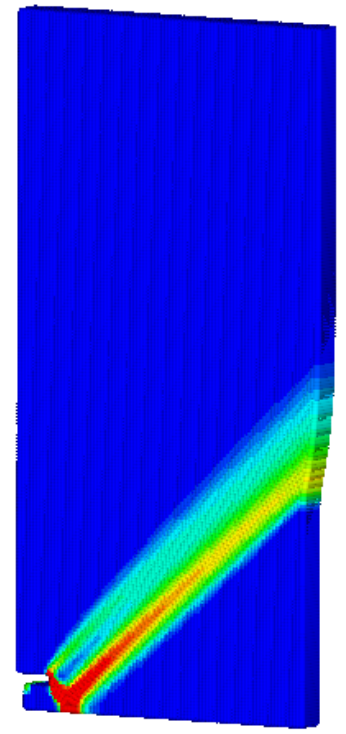

d) $0.5 \mathrm{~ms}$

Figure 8. Effective plastic strain of a specimen subjected to tensile loading at different time steps for the 180,000 particle discretization

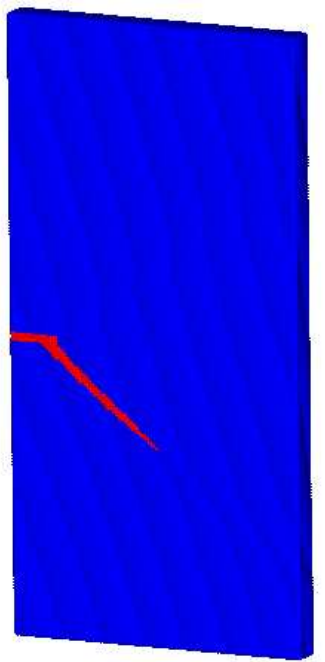

a) $0.1 \mathrm{~ms}$

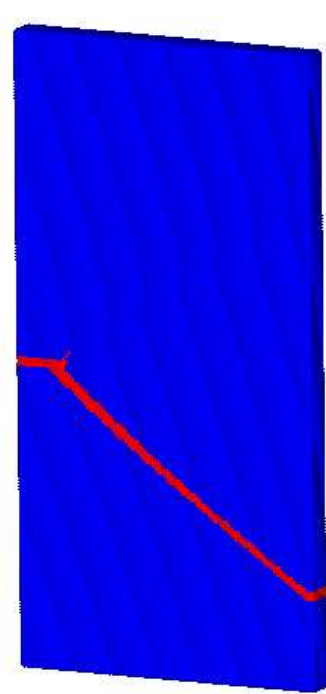

b) $0.16 \mathrm{~ms}$

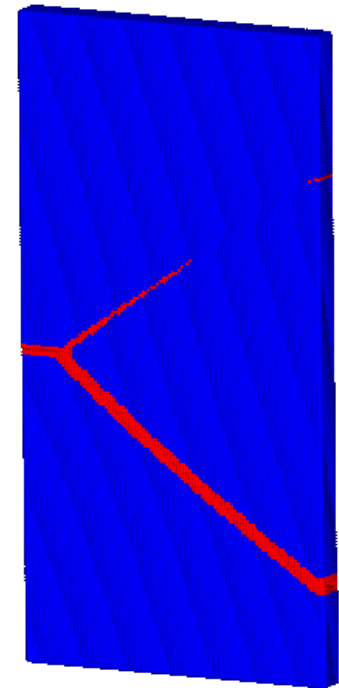

c) $0.18 \mathrm{~ms}$

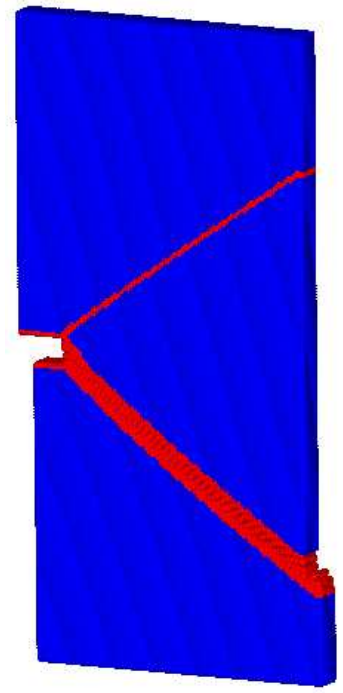

d) $1.0 \mathrm{~ms}$

Figure 9. Deformation of a specimen subjected to tensile loading at different time steps 


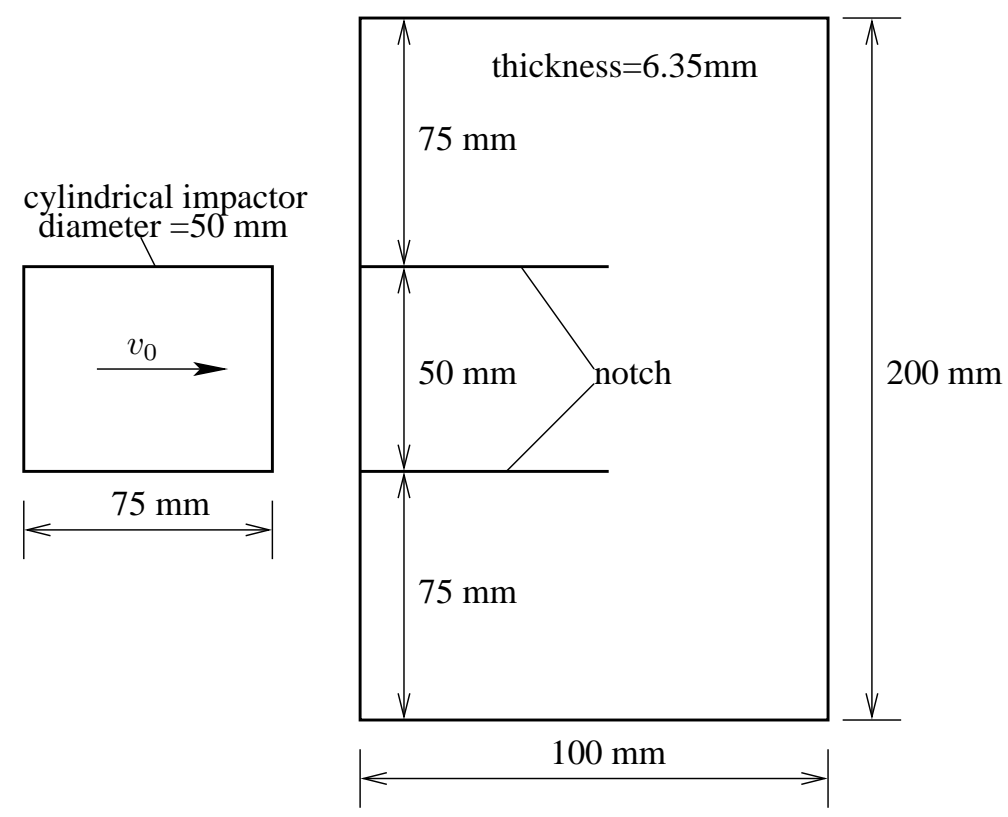

Figure 10. The Kalthoff problem: test setup

Cook model is given by

$$
\sigma_{Y}=\left(A+B \gamma^{n}\right)\left(1+C \ln \dot{\epsilon}^{*}\right)\left(1-T^{* m}\right)
$$

with $\dot{\epsilon}^{*}=\dot{\gamma} / \dot{\gamma}_{0}$ where $\gamma$ is the effective plastic strain and $\dot{\gamma}_{0}$ is the reference strain rate taken to be $1.0 / \mathrm{s}$ and

$$
T^{*}=\frac{T-T_{r}}{T_{m}-T_{r}}
$$

where $T_{r}$ is the reference temperature and $T_{m}$ is the melting temperature. The material parameters are $A=792 M P a, B=509 M P a, C=0.014, n=0.26, m=0.55$. The density is $\varrho=7800 \mathrm{~kg} / \mathrm{m}^{3}$, bulk modulus $K=157 \mathrm{GPa}$, shear modulus $G=76 \mathrm{GPa}$ and the specific heat is $c_{v}=477 \mathrm{~J} / \mathrm{kgC}$, reference temperature $T_{r}=296 \mathrm{~K}$ and melting temperature $T_{m}=1033 \mathrm{~K}$. We used a Quinney factor $\beta=0.9$ for the temperature update:

$$
\Delta T=\int_{0}^{\gamma} \frac{\beta}{\varrho c_{v}} \sigma_{Y} d \gamma
$$

The behavior at each material point is governed by both a Rankine criterion (with $f_{t}=2 A$ ) and the material stability criterion. The onset of a material instability is then determined by whichever criterion indicates loss of material stability first. When the Rankine criterion is met, a cracked particle is introduced as in Rabczuk and Belytschko [25, 26]. If the $J_{2}$ stability criterion is triggered, a sheared particle is introduced (it appears to be a natural consequence of the acoustic tensor for $J_{2}$ pasticity that $\mathbf{h}$ is approximately perpendicular to $\mathbf{n}$ ). We used two 
different particle arrangements, with approximately 140,000 particles and 1,035,000 particles, respectively. The impactor is also discretized.

First, we present the results with an impact velocity of $60 \mathrm{~m} / \mathrm{s}$. The shear band pattern for the 140,000 particle computation at different time steps is shown in figure 11. We are able to capture the principal shear band pattern observed in the experiment. The results for the 1,035,000 particle simulation are very similar to the results obtained by the coarse model.

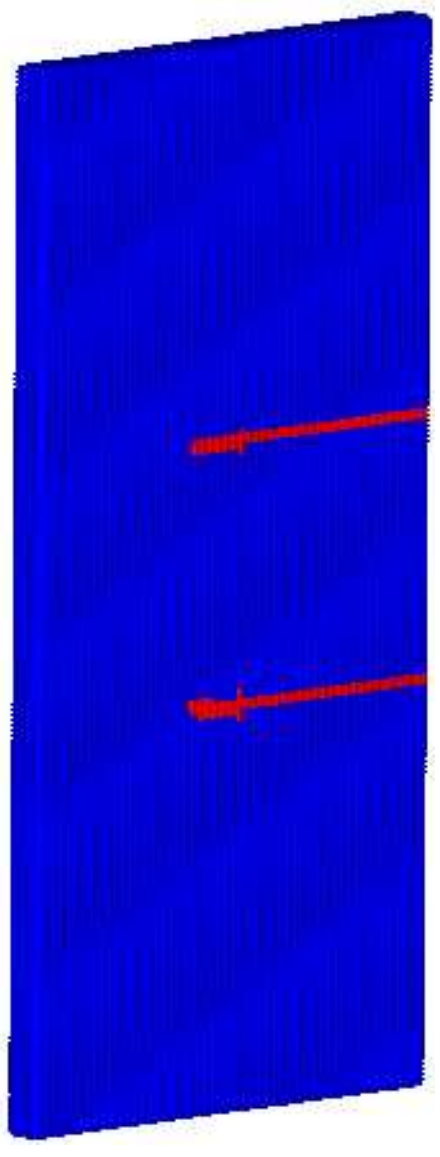

a)

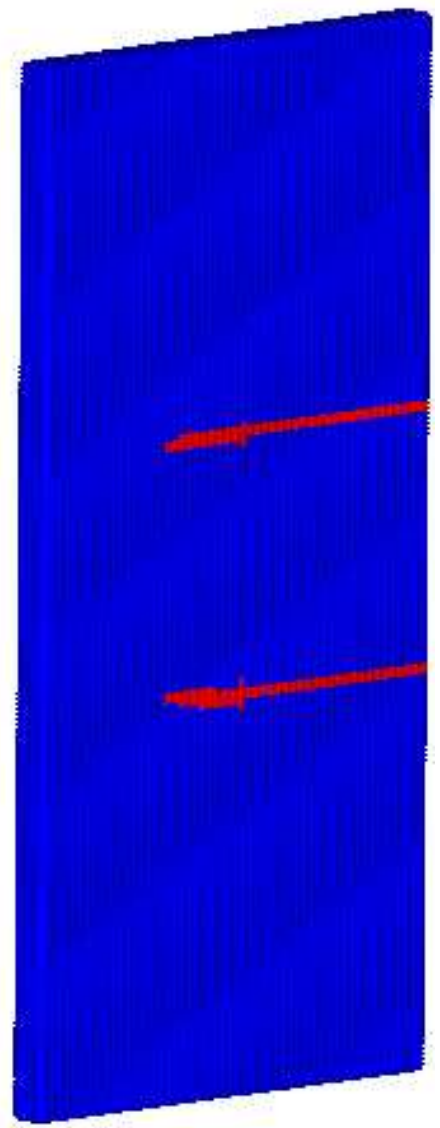

b)

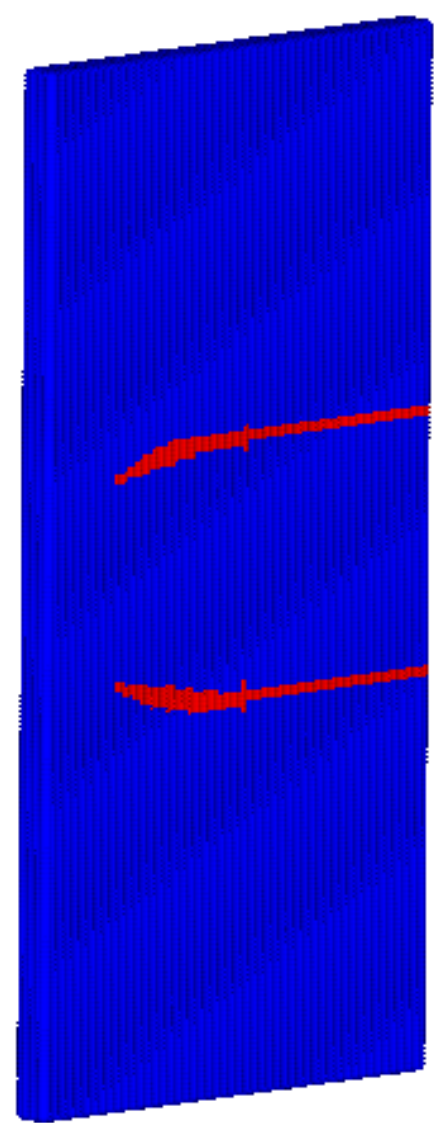

c)

Figure 11. Shear band for the 140,000 particle discretization for the Kalthoff problems at different time steps and an impact velocity of $60 \mathrm{~m} / \mathrm{s}$; ductile failure occurs

Results for an impact velocity of $40 \mathrm{~m} / \mathrm{s}$ are shown in figure 12 ; failure is still predicted by a ductile mode. Figure $12 \mathrm{~d}$ shows the effective plastic strains while figures $12 \mathrm{a}-\mathrm{c}$ show the shear band, i.e. the failed particles. As can be seen from figures 12 , the shear band is arrested midway to the left edge. This behavior and the curvature of the shear bands correspond well to that observed by Kalthoff and Winkler [44] and Kalthoff [45].

The simulation at an impact velocity of $20 \mathrm{~m} / \mathrm{s}$ correctly predicts the transition to brittle failure, see figure 13. The crack propagates with an angle of approximately 70 degrees. In figure 


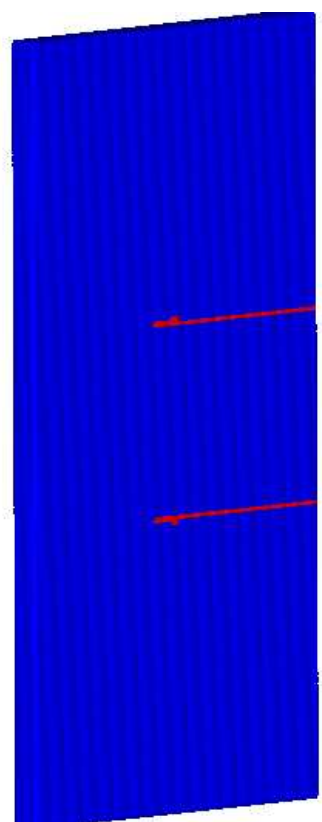

a)

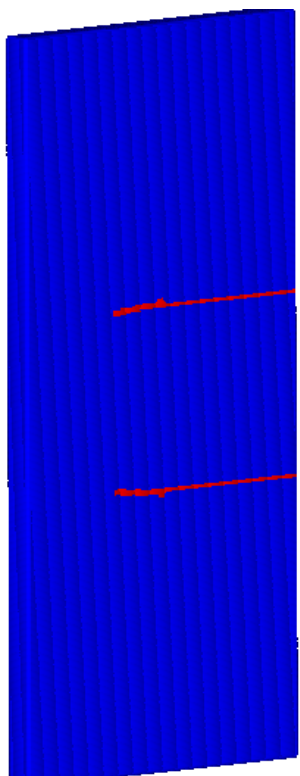

b)

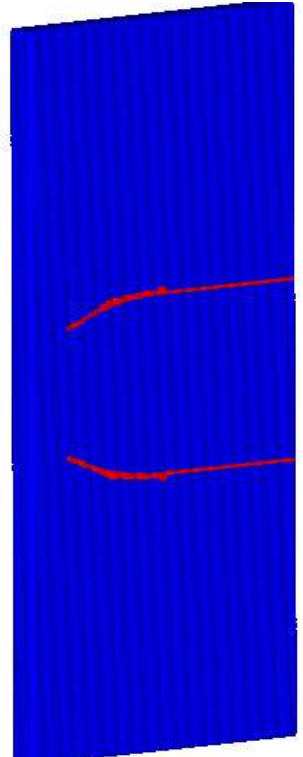

c)

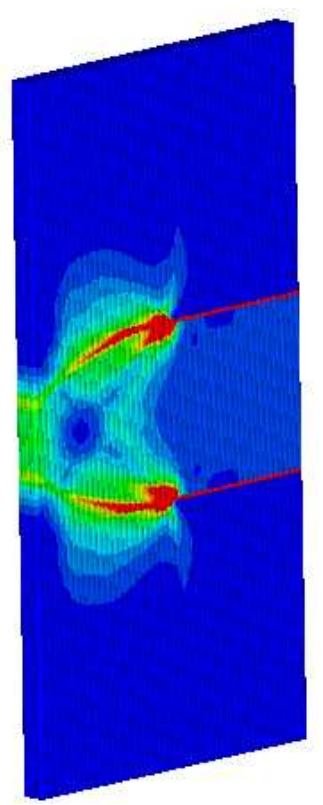

d)

Figure 12. a)-c)Shear band evolution for the 1,035,000 particle discretization for the Kalthoff problems at different time steps and an impact velocity of $40 \mathrm{~m} / \mathrm{s}$; a ductile failure occurs,d) corresponding effective plastic strain distribution

13: we show only the results for the 1,035,000 particle discretization. The results for the coarse discretization look almost identical. The crack pattern is similar to the one of the experiment. Some oscillations in the crack paths can be observed. The crack speed is shown in figure 14. The Rayleigh wave speed of approximately $2800 \mathrm{~m} / \mathrm{s}$ is not exceeded.

\subsection{Plate with two holes}

Consider a plate with two holes under compression as shown in figure 15. This example was studied by Diez et al. [52] using a viscoplastic constitutive model. They modified the location of the holes and discovered that it altered the shear band patterns. Here a structured model was used. The two holes are generated by a technique described in Rabczuk and Belytschko [53]. The compression load is applied as a velocity boundary condition, with a constant velocity of $1 \mathrm{~m} / \mathrm{s}$ applied on the top.

In contrast to Diez et al. [52], we use an elasto-plastic model from Areias [54]. The model is similar to the viscoplastic model in [52] and as we will see, we are able to capture the principal failure mechanisms as in [52]. The material parameters are Young's modulus $E=200 G P a$, Poisson's ratio $\nu=0.3$, initial yield strength $\sigma_{0}=200 M P a$ and softening modulus $H=-20 G P a$. We consider a coarse model and a fine model discretized with approximately 20,000 and 80,000 particles, respectively. We first discuss the results where the holes are separated by $10 \mathrm{~mm}$ in the horizontal direction, see LHS of figure 15 . 


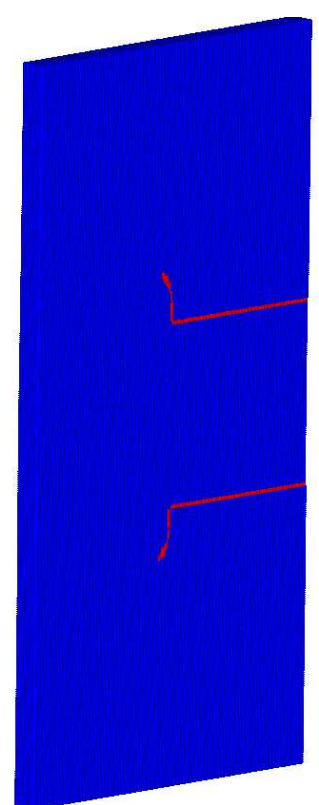

a)

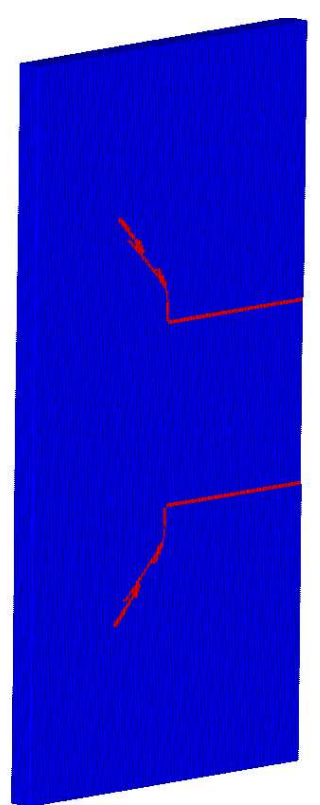

b)

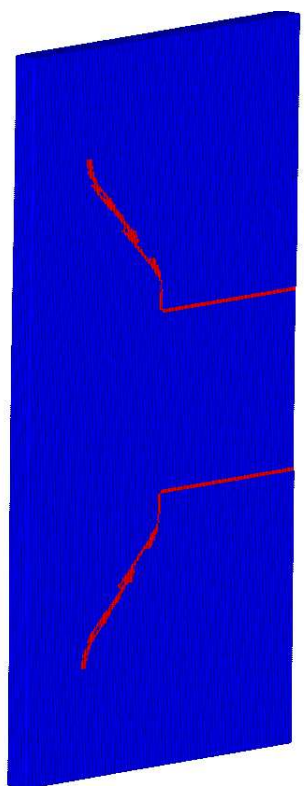

c)

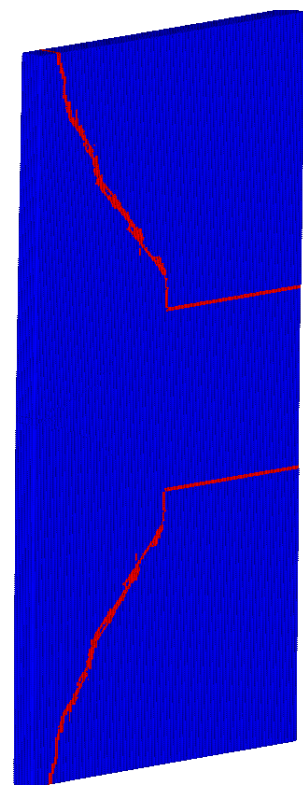

c)

Figure 13. Crack evolution for the 1,035,000 particle discretization for the Kalthoff problems at different time steps for an impact velocity of $20 \mathrm{~m} / \mathrm{s}$; a brittle failure occurs

The shear band pattern at different time steps and the effective plastic strains at the end of the computation are shown in figures 16 and 17 for the two models. The holes almost closed during the course of the computation. The holes appear as black circles. The progression of the shear band from the two holes at the beginning of the computation can clearly be seen. The two holes are completed connected at $1.4 \mathrm{~ms}$. At $1.7 \mathrm{~ms}$ the specimen is sheared completely into two pieces. Due to the relatively large distance in $x$-direction between the two holes, only a single shear band develops.

The load displacement curves for the two discretizations is shown in figure 18 and don't show mesh dependence. Though the constitutive model is different from the one in [52], the load deflection curve looks very similar to the one in [52].

Next, we consider the example as shown on the RHS of figure 15. Diez et al. [52] reported that a very fine mesh was necessary to capture the correct shear band paths. Their mesh was adaptively refined across the crack path. We use the fine structured models as mentioned above. The final shear band pattern with the corresponding effecitve plastic strain distribution is shown in figure 19. As in [52], two parallel shear bands occur. The main difference between our results and the results in [52] is that in [52], a shear band connected the two holes. In our simulation, two shear band connect the lower shear band with the upper shear band close to the notch. 


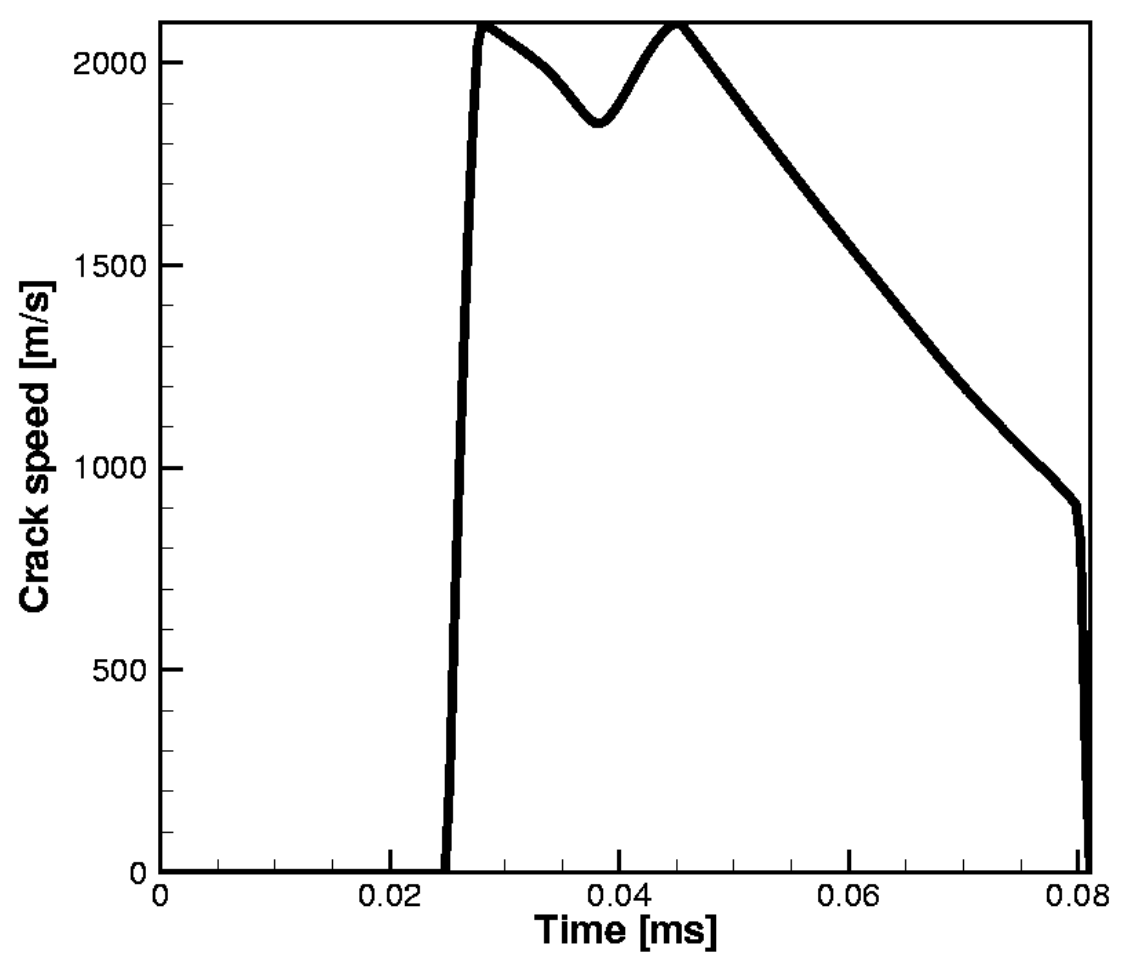

Figure 14. Crack speed for the Kalthoff problem for a brittle failure mode

\subsection{Plane strain indentation}

This example shows the performance of our method for curved shear bands with intersections. Consider the plane strain indentation problem as illustrated in figure 20. The specimen is held in the horizontal and vertical directions at the bottom and in the horizontal direction at the sides. The vertical displacement under the punch is prescribed to increase linearly with time. The loading plate is modelled by finite elements. This example was also studied by Miehe and Lambrecht [55].

The material parameters are: bulk modulus $K=160,000 M P a$; shear modulus $G=$ 80, 000MPa; yield stress $\sigma_{Y}=500 M P a$ and softening modulus $H=-131 M P a$. As in [55], we used a $J 2$ plasticity model. We studied 3 different discretizations, 12,000 particles, 30,000 particles and 120,000 particles.

The deformed specimen at different times for the 30,000 particle discretization is shown in figure 21. The shear band pattern looks very similar to the shear band pattern in [55]. In contrast to [55] who performed his computation up to a maximum vertical top displacement of $1.8 \mathrm{~mm}$; we carried out the simulation to a later stage to $4 \mathrm{~mm}$ vertical top displacement. At that time, another shear band pattern appears as shown in figure 21e. Four larger fragments are formed that start to separate from the specimen. The load deflection curve is shown in 


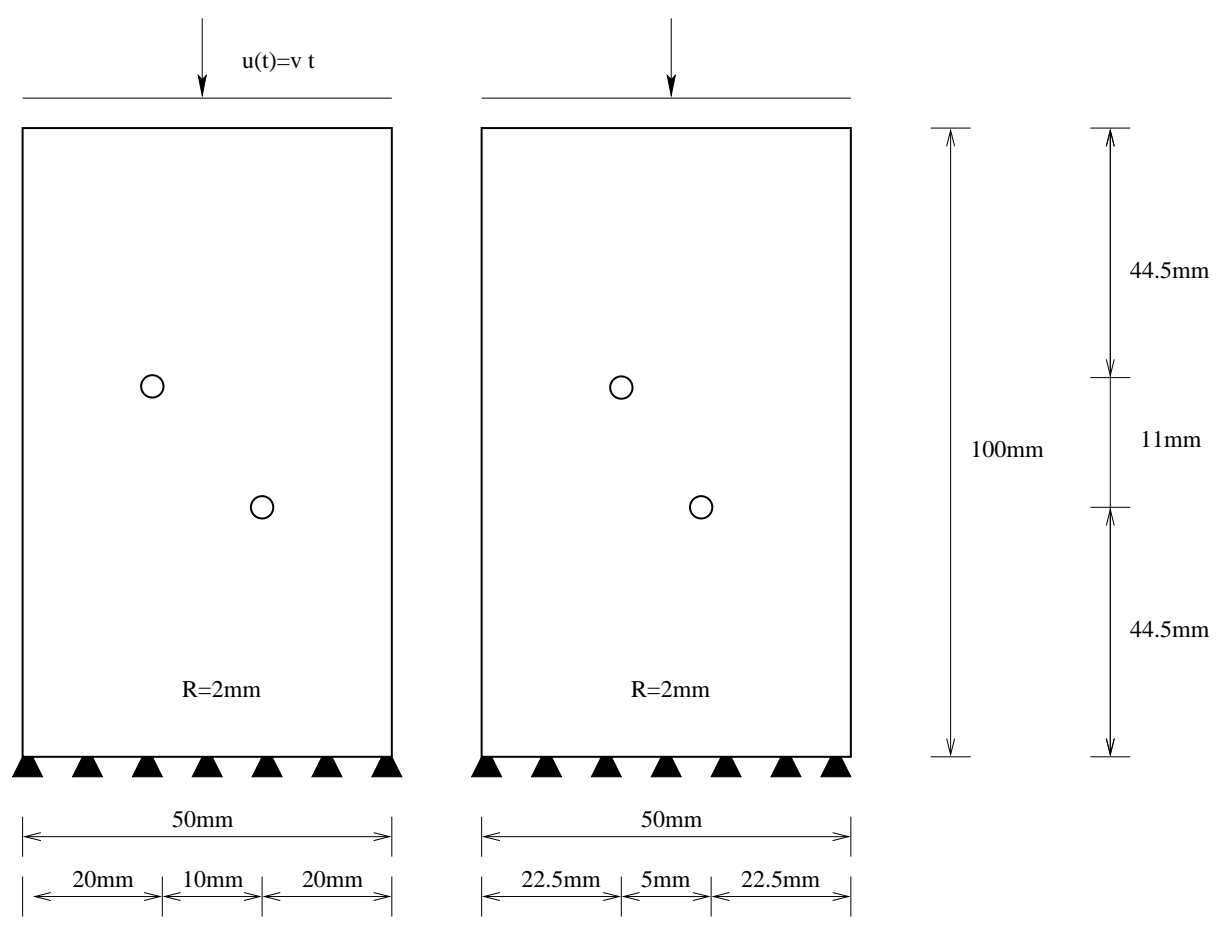

Figure 15. Model description for the plate with two holes under compression

figure 22, It is similar to [55] and also does not show mesh dependence.

\subsection{The Nesterenko experiment}

Nesterenko et al. [56], Xue et al. [57], Meyers et al. [58], Shih et al. [59] performed a series of multiple shear banding experiments in cylinders for different type of materials and different dimensions. The test-set up is shown in figure 23. In all experiments, the cylindrical test specimen is surrounded by a copper stopper tube and a copper driver tube, that is itself surrounded by an explosive. We will consider here the titanium test specimen and the stainless steel specimen. We carried out two dimensional computations and modelled also the explosive. The density of the explosive is $0.9 \mathrm{~g} / \mathrm{cm}^{3}$ and the detonation velocity is according to Xue et al. [57] $4000 \mathrm{~m} / \mathrm{s}$. They also measured the initial velocity of the inner wall of the tube $(200 \mathrm{~m} / \mathrm{s})$ that can be used for the numerical simulation. We will show results for a discretization with 60,000 particles under the assumption of plain strain conditions.

As for the Kalthoff problem, we use the Johnson-Cook model for copper. We assume that the plastic work is completely transformed into heat. The material data for copper are: density 


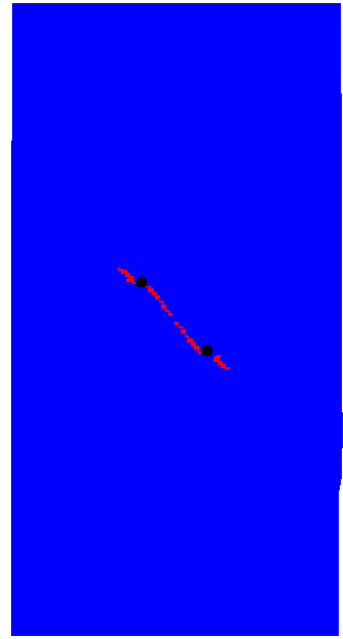

a) $1.0 \mathrm{~ms}$

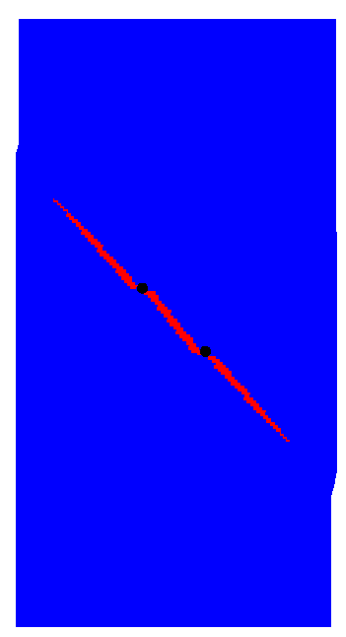

b) $1.4 \mathrm{~ms}$

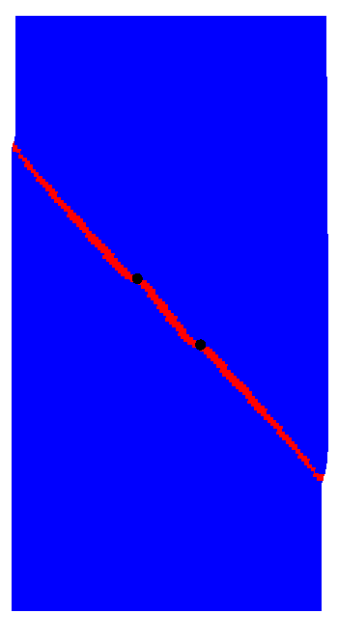

c) $1.7 \mathrm{~ms}$

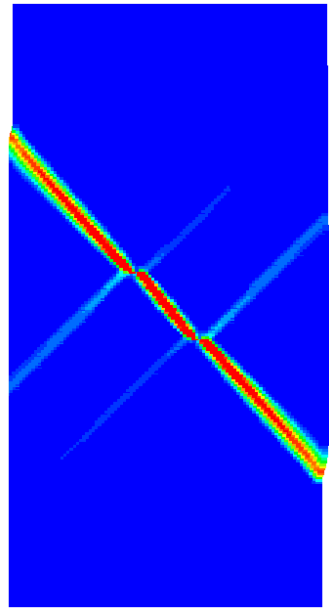

d) $1.7 \mathrm{~ms}$

Figure 16. a)-c) Sheared particles and d) effective plastic strain of the plate with two holes at different time steps for 20,000 particles

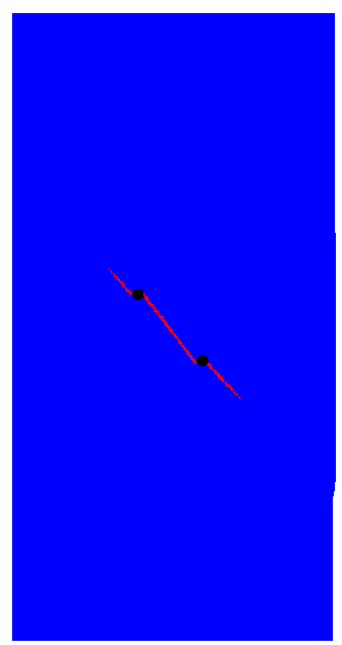

a) $1.0 \mathrm{~ms}$

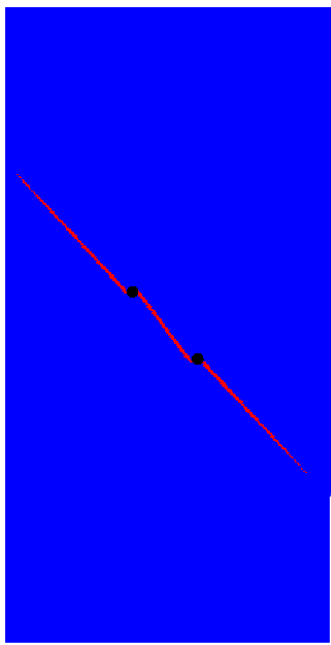

b) $1.4 \mathrm{~ms}$

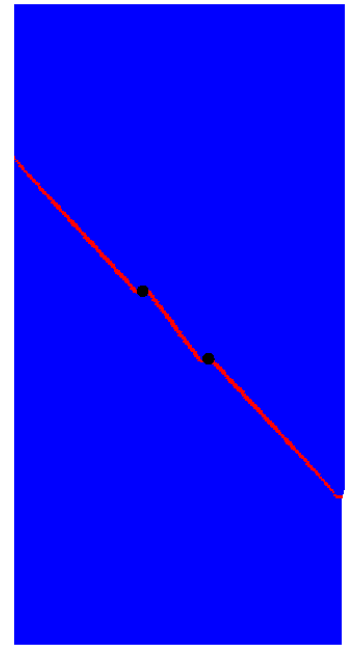

c) $1.7 \mathrm{~ms}$

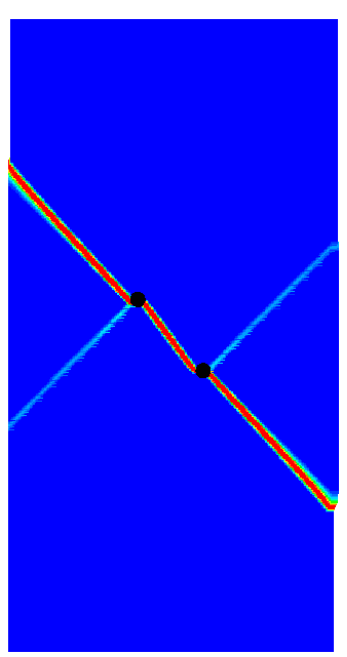

d) $1.7 \mathrm{~ms}$

Figure 17. a)-c) Sheared particles and d) effective plastic strain of the plate with two holes at different time steps for 80,000 particles 


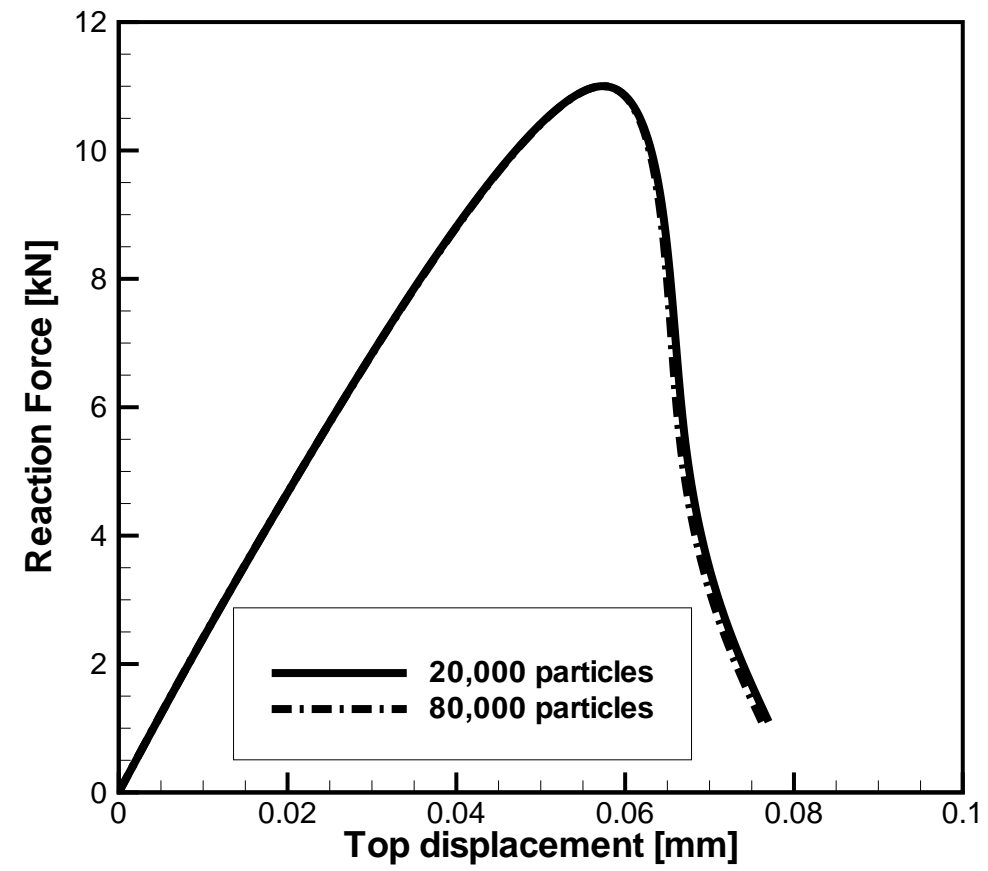

Figure 18. Load deflection curve for the plate under compression with 2 holes

$\varrho=8.92 \mathrm{~g} / \mathrm{cm}^{3}$, bulk modulus $K=115 G P a$, shear modulus $G=44 G P a$, melting temperature $T_{m}=1058 K, A=90 M P a, B=292 M P a, n=0.31, C=0.025, m=1.09$; specific heat $c_{v}=385 \mathrm{~J} / \mathrm{kg} \mathrm{K}$. For stainless steel, the material parameters are density $\varrho=7.9 \mathrm{~g} / \mathrm{cm}^{3}$, bulk modulus $K=200 G P a$, shear modulus $G=77.5 \mathrm{GPa}$, melting temperature $T_{m}=1396 \mathrm{~K}$, $A=110 \mathrm{MPa}, \mathrm{B}=1500 \mathrm{MPa}, n=0.36, C=0.014, \mathrm{~m}=1$ and specific heat $c_{v}=500 \mathrm{~J} / \mathrm{kg} \mathrm{K}$. In some computations we have randomly varied the material yield strength spatially within the specimen. For this purpose, we multiplied the material strength with a factor $0.75<\alpha<1.25$, obtained from a log-normal distribution around the mean value of 1 with a standard deviation of $5 \%$. The intent is to model defects or imperfections of the material. The shear band pattern will also depend on the spatial correlation of these imperfections. For the examples we tested, we obtained similar shear band patterns, although the number of shear bands decreased with increase in the scatter of the material properties.

For titanium, Nesterenko et al. [56] used the following for the yield surface:

$$
\sigma=\sigma_{0}\left(1-a\left(T-T_{0}\right)\right)\left(\frac{\dot{\epsilon}}{\dot{\epsilon}_{0}}\right)^{m}
$$

where $\sigma_{0}$ is the flow stress, $T_{0}$ is the reference temperature, $\dot{\epsilon}_{0}$ is a reference strain rate, $a$ is a softening parameter and $m$ is the so-called strain rate sensivity. The material parameters 


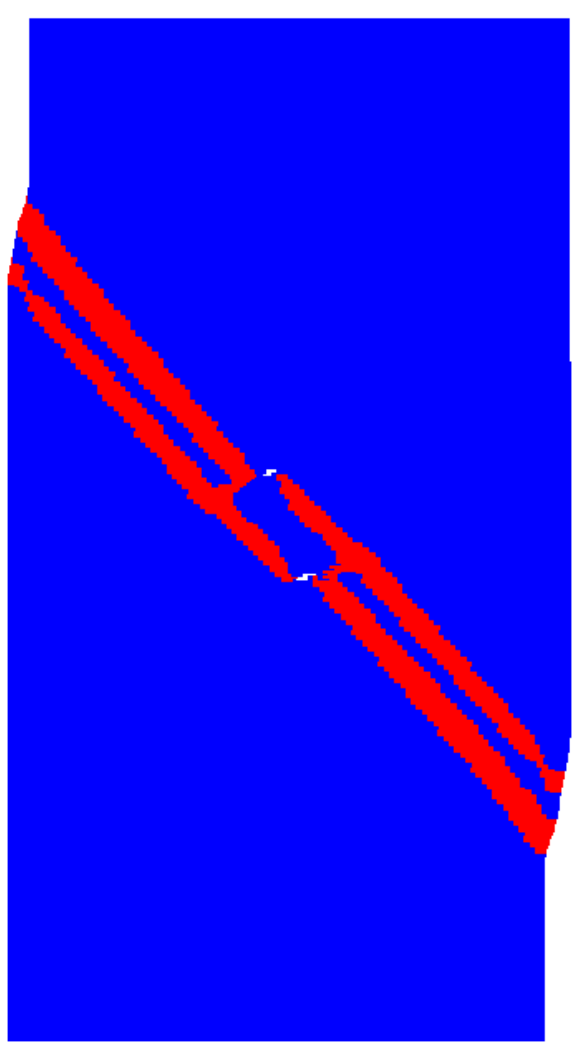

a)

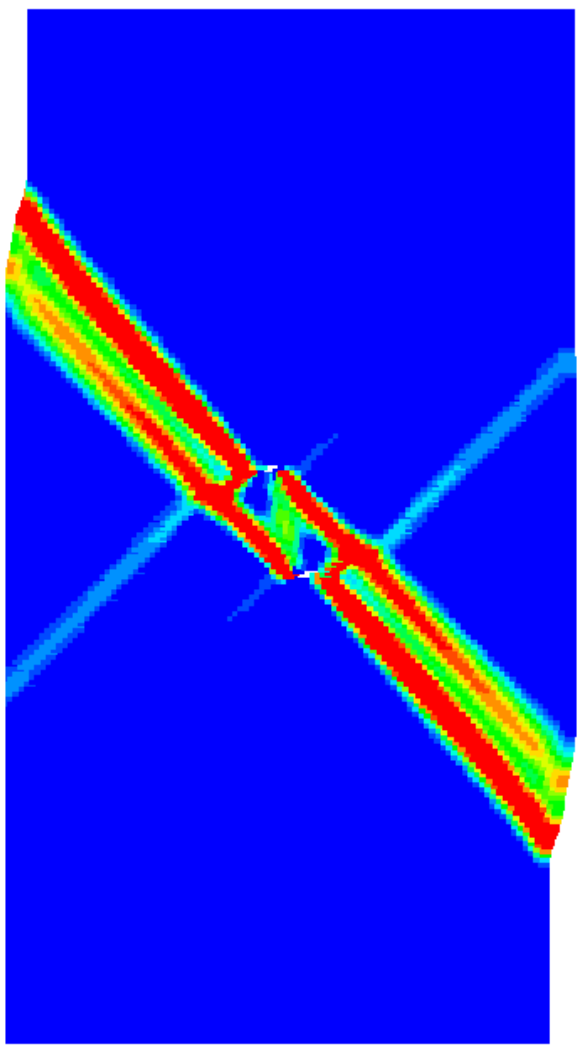

b)

Figure 19. a) Shear band pattern and b) effective plastic strain of the 2-hole-problem

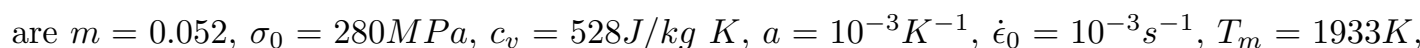
density $\varrho=4.5 \mathrm{~g} / \mathrm{cm}^{3}$, Young's modulus $E=10.85 \mathrm{GPa}$ and Poisson ratio $\nu=0.34$; we used the same parameters.

Figure 24 shows the shear band pattern near the beginning and at the end of the computation for titanium. As can be seen from figure 24, initially, a large number of shear bands are nucleated. However, a few of the shear bands become dominant in the later stages of the evolution. One of the experimental results from Nesterenko et al. [56] is depicted in figure 24c. Comparison with our computations shows many noteworthy similarities in the patterns and also some descrepancies. As in the experiment, a few shear bands become dominant in the later stages growth. However, in the experiment, some shear bands grow at an opposite angle with the radial direction, whereas this did not occur in the computation. Moreover, the final tangential direction of the shear bands is smaller in the experiment than in the computation. This may be due to inadequancies in our material model or due to the assumption of perfect cylindrical symmetry in the computational load; the senior author (T. Belytschko) notes that it is very difficult to achieve cylindrical symmetry in explosive loadings.

We repeated the computations with smaller scatter in the material properties. The results 


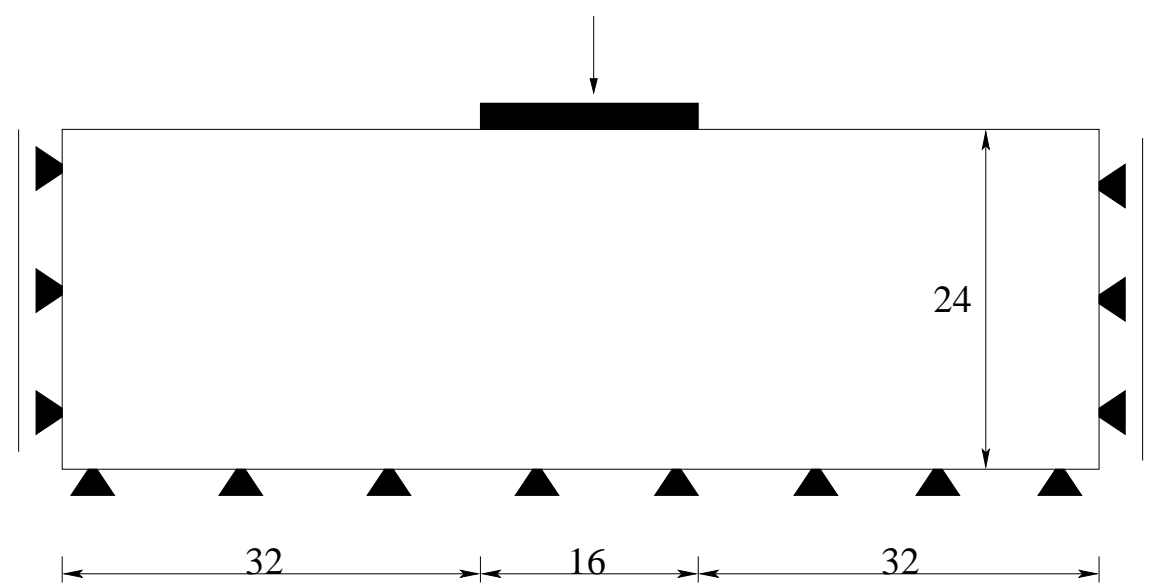

Figure 20. Set-up of the plane strain indentation problem

are not shown here; we found that as the scatter decreases, the number of dominant shear bands increase, but their length decreases. Thus the interplay of material imperfections and material instability plays a critical role in the final pattern.

The shear band pattern of the steel specimen is shown in figure 25. Much less shear banding occurs here because the onset of softening requires larger strains. The shear band pattern also agrees well with the experimental results of Nesterenko et al. [56].

\section{CONCLUSIONS}

We have presented a nonlinear meshfree method applicable to material and geometric nonlinearities for modelling shear bands with cohesive surfaces. The shear band is modelled by a set of sheared particles. In each sheared particle, a discontinuity in the tangential component of the displacement is added to the displacement field. The method is a variation of the theme "cracking particles" [25]. It is very easy, as shown in the computation of the Kalthoff example, to include cracking and sheared particles in a single computation to model complex failure processes.

The method was applied to several problems for which experiments or previous computations were available. It is noteworthy that:

1. The method reproduced the brittle/ductile transition observed in the Kalthoff experiments.

2. The method is able to provide reasonably good reproductions of the complex shear band patterns found in the Nesterenko et al. [56] experiments.

The methodology as described occasionally encounters difficulties when shear bands intersect. This requires the addition of an enrichment for intersecting discontinuities, as described in Belytschko et al. [60]. In addition, the propagation of a shear band through 


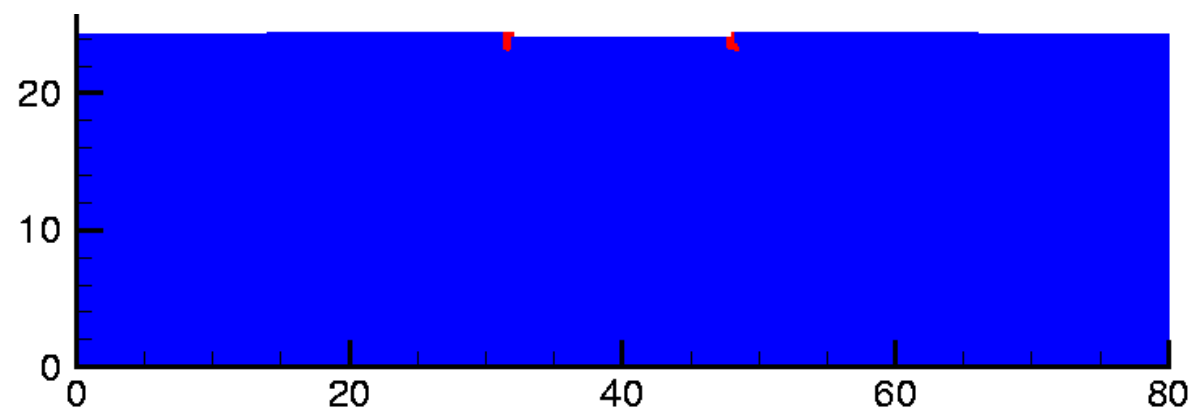

a) $0.24 \mathrm{~mm}$

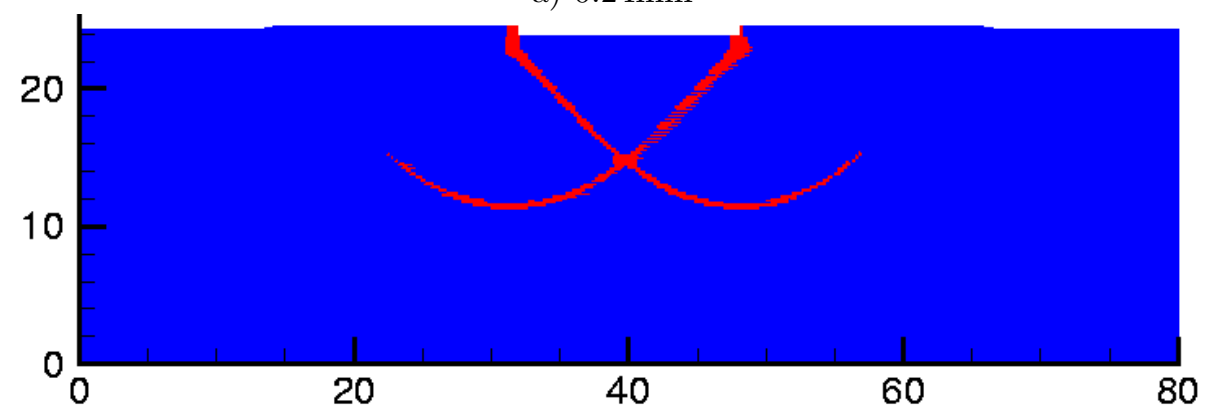

b) $0.56 \mathrm{~mm}$

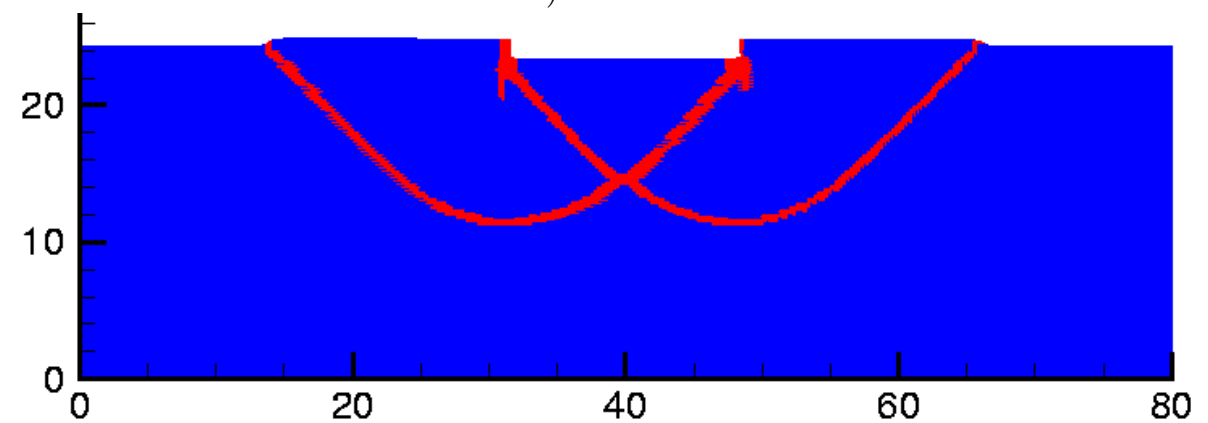

c) $1.04 \mathrm{~mm}$

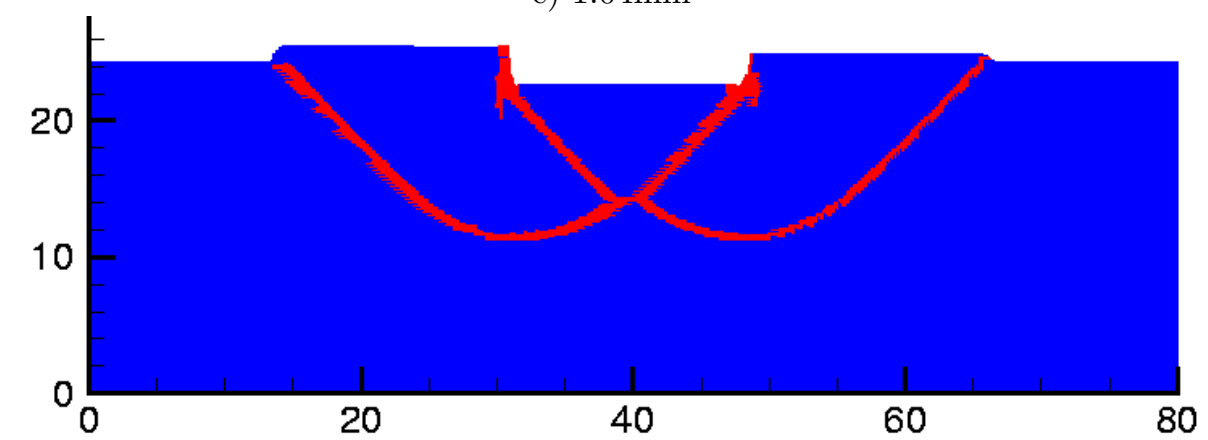

d) $1.64 \mathrm{~mm}$

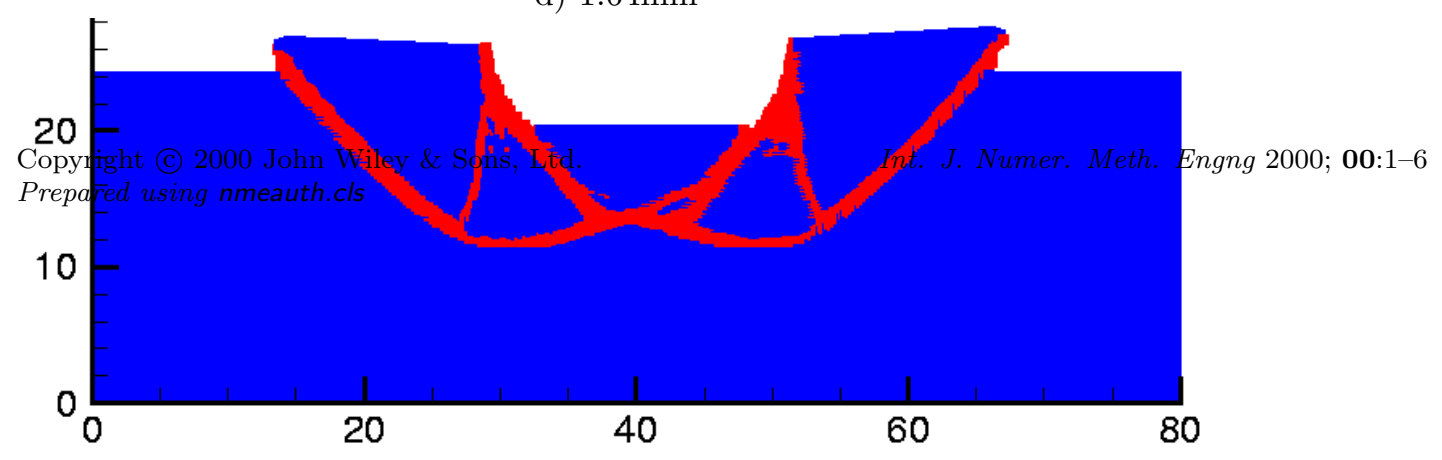




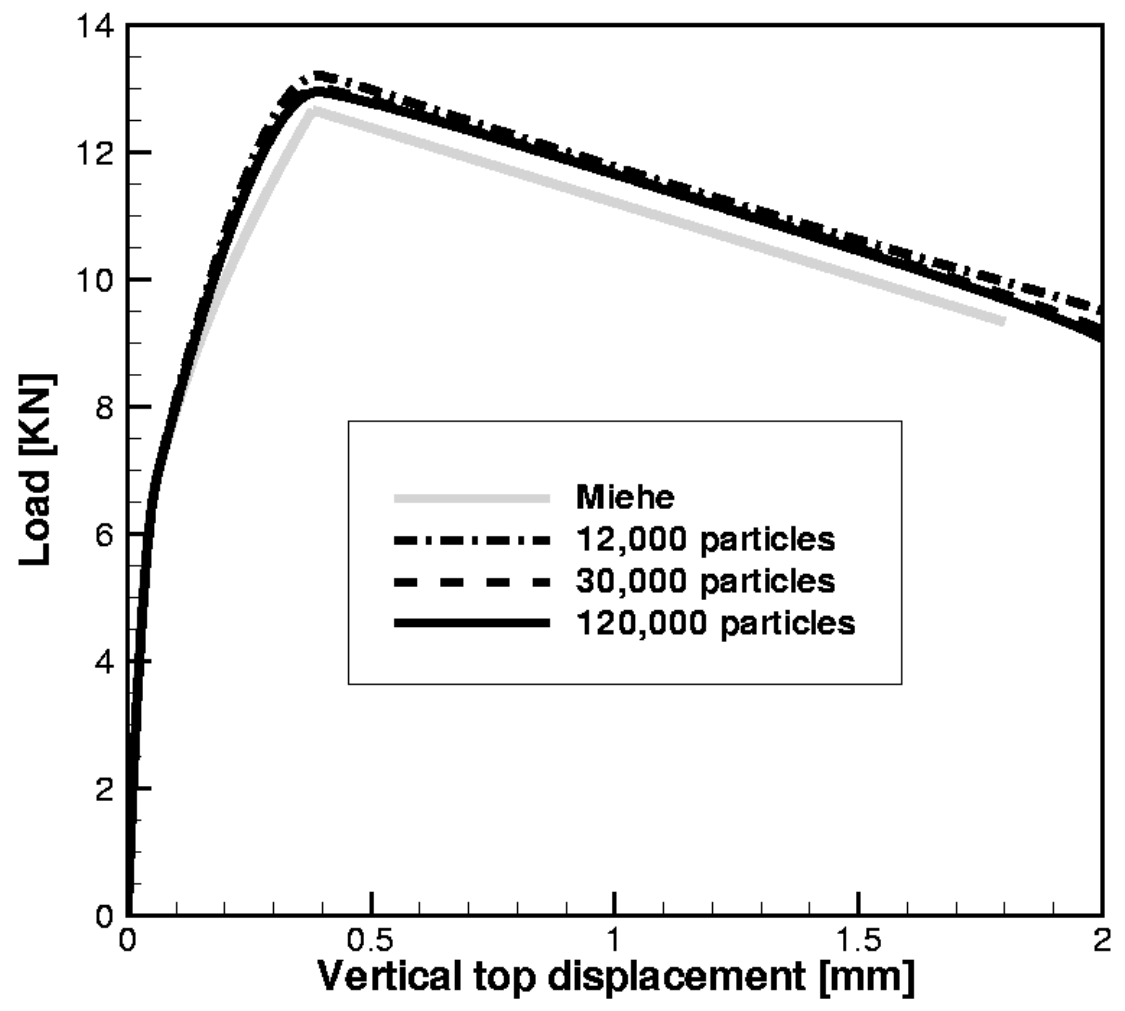

Figure 22. Load deflection curve for the indentation problems copper driver tube

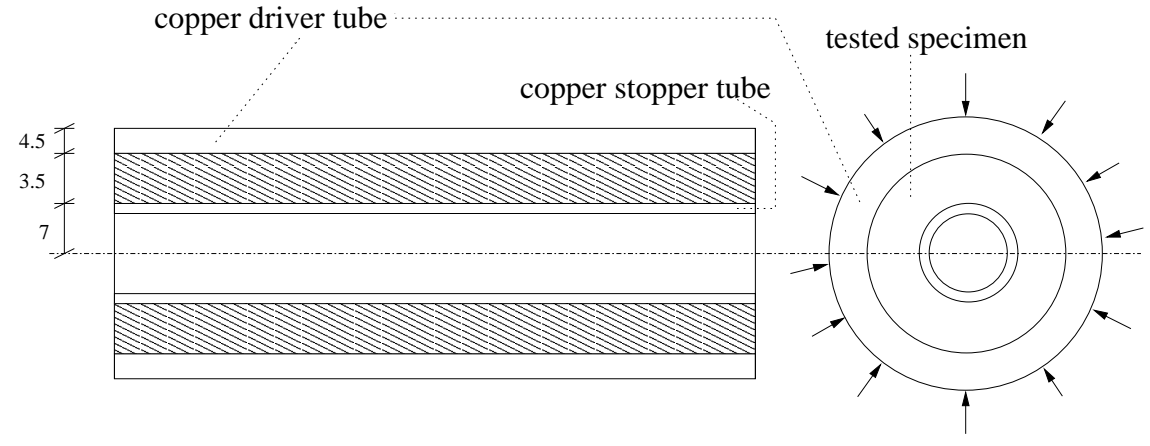

Figure 23. Typical test-setup of the Nesterenko et al. [56] experiment, dimensions in mm

an existing shear band poses problems because hyperbolicity has already been lost at the intersection.

The methodology is not guaranteed to achieve completeness in the tangential displacement, 


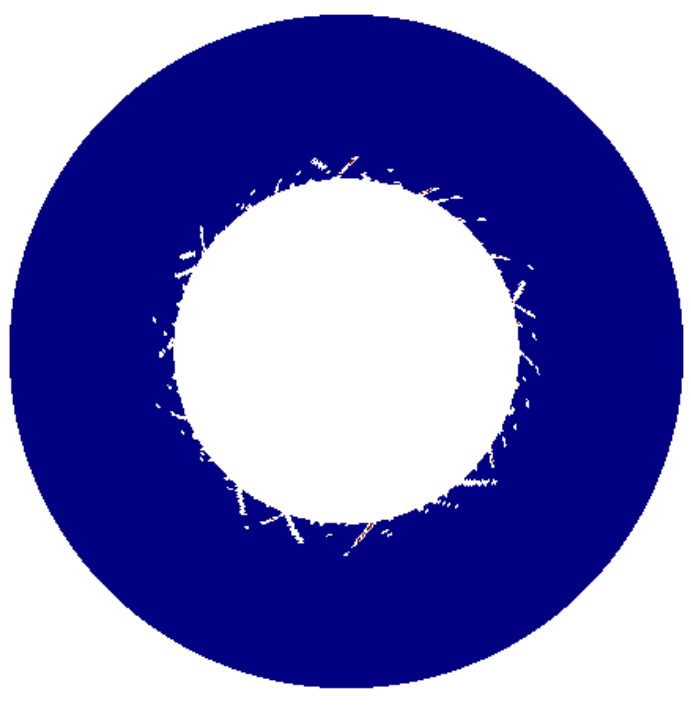

(a) early stage

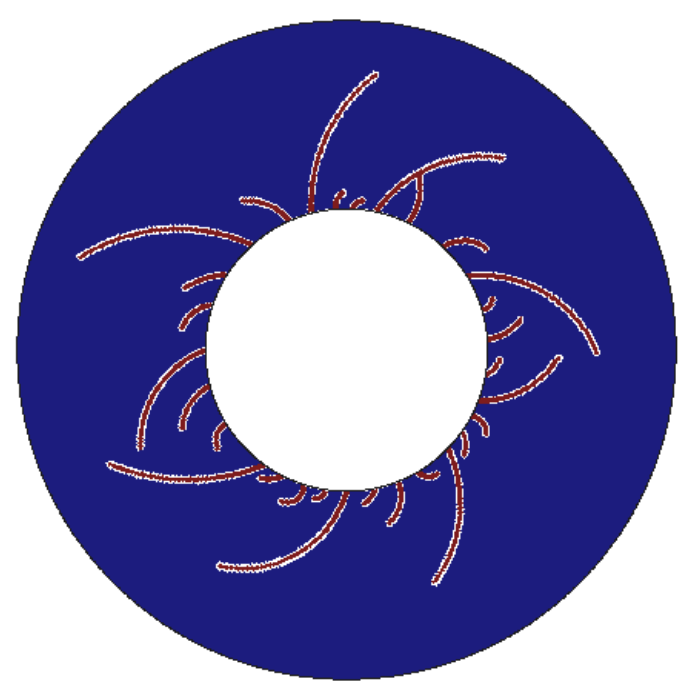

(b) final stage

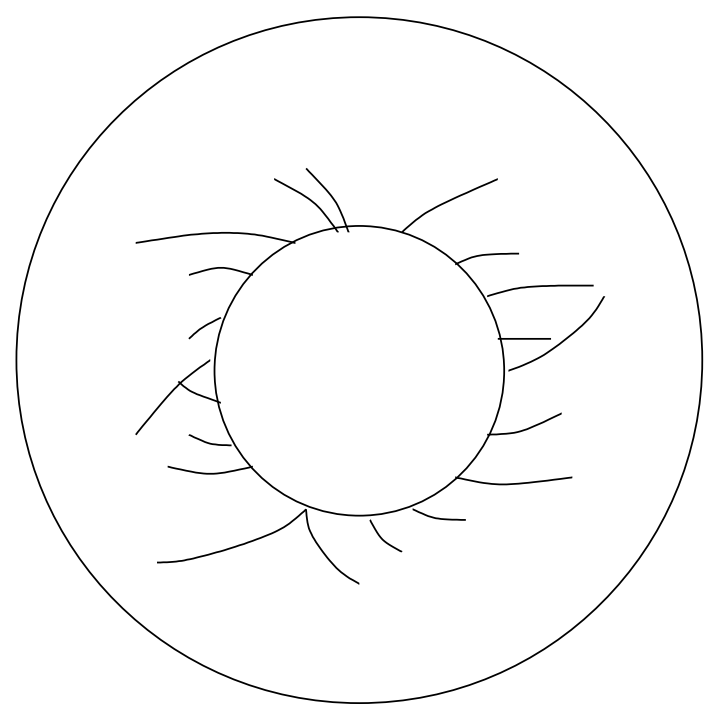

(c) experiment

Figure 24. Shear band formation at different load steps for the Nesterenko et al. [56] experiment; titanium specimen 


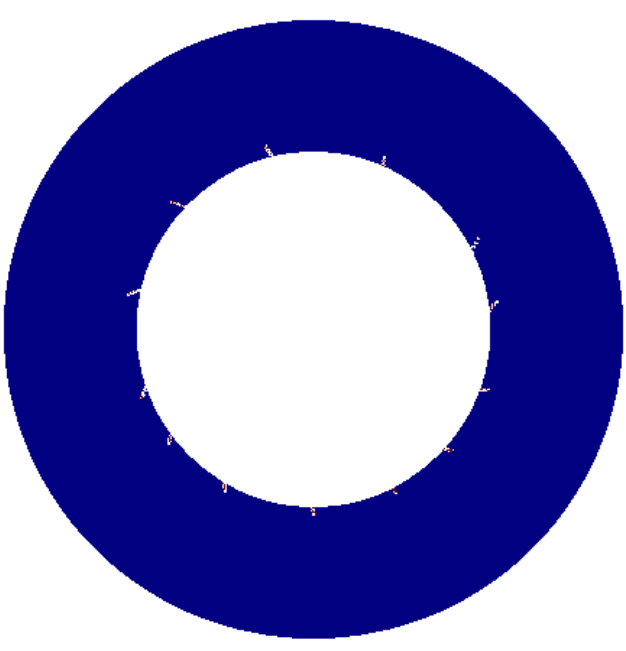

a)

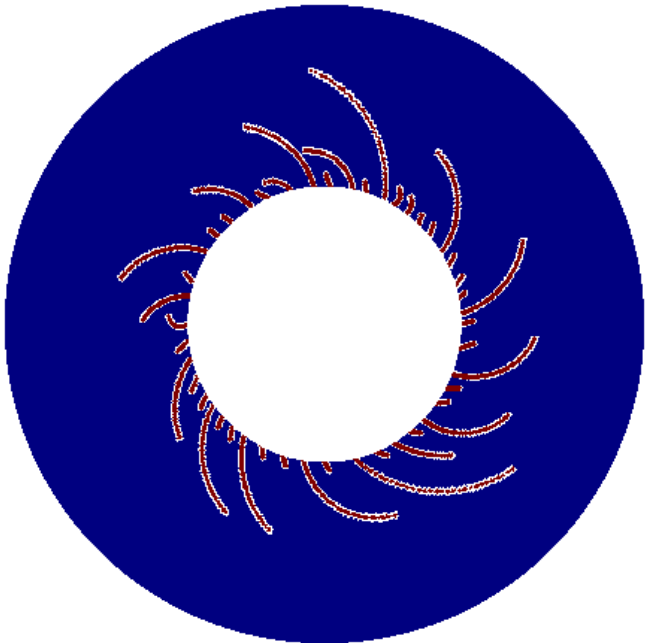

b)

Figure 25. Shear band formation at different load steps for the Nesterenko et al. [56] experiment; stainless steel specimen

i.e. the capability to represent constant and linearly varying jumps in the tangential direction. Lack of completeness may arise from an insufficient number of sheared particles in the shear band path. This could be corrected by an adaptive procedure such as in [26] by adding particles in the subdomain around where material instability is incipient.

The method is based on a Lagrangian kernel, so spurious tensile instabilities often found in particle methods do not arise. The attractive feature of the method is the simplicity of treating complicated patterns of shear bands. It is only necessary to identify the nodes at which the material has lost stability. The kinematics of the shear band are then completely treated by adding an additional displacement mode at each node. This feature is easily added to particle methods and provides a method of attractive simplicity. 


\section{ACKNOWLEDGEMENT}

The support of Office of the Naval Research under Grant N00014-03-1-0097 and the Army Research Office under Grant DAAD19-02-01-0339 is gratefully acknowledged.

references

[1] Z. P. Bazant and T. Belytschko. Wave propagation in a strain-softening bar: Exact solution. Journal of Engineering Mechanics-ASCE, 111(3):381-389, 1985.

[2] D. Lasry and T. Belytschko. Localization limiters in transient problems. International Journal of Solids and Structures, 24(6):581-597, 1988.

[3] A. Needleman and V. Tvergaard. Analysis of brittle-ductile transition under dynamic shear loading. International Journal of Solids and Structures, 32:2571-2590, 1995.

[4] M. Jirasek. Comparative study on finite elements with embedded discontinuities. Computer Methods in Applied Mechanics and Engineering, 188:307-330, 2000.

[5] X.-P. Xu and A. Needleman. Numerical simulations of fast crack growth in brittle solids. Journal of the Mechanics and Physics of Solids, 42:1397-1434, 1994.

[6] G. T. Camacho and M. Ortiz. Computational modeling of impact damage in brittle materials. International Journal of Solids and Structures, 33:2899-2938, 1996.

[7] M. Ortiz, Y. Leroy, and A. Needleman. Finite element method for localized failure analysis. Computer Methods in Applied Mechanics and Engineering, 61(2):189-214, 1987.

[8] F. Zhou and J.F. Molinari. Dynamic crack propagation with cohesive elements: a methodolgy to address mesh dependence. International Journal for Numerical Methods in Engineering, 59(1):1-24, 2004.

[9] Q. Yang, A. Mota, and M. Ortiz. A class of variational strain-localization finite elements. International Journal for Numerical Methods in Engineering, 62(8):1013-1037, 2005.

[10] M.L. Falk, A. Needleman, and J.R. Rice. A critical evaluation of cohesive zone models of dynamic fracture. Journal of Physics IV 11 (PR5), pages 43-50, 2001.

[11] N. Moes, J. Dolbow, and T. Belytschko. A finite element method for crack growth without remeshing. International Journal for Numerical Methods in Engineering, 46(1):133-150, 1999.

[12] N Moes and T. Belytschko. Extended finite element method for cohesive crack growth. Engineering Fracture Mechanics, 69:813-834, 2002.

[13] A. Gravouil, N. Moes, and T. Belytschko. Non-planar 3D crack growth by the extended finite element and level sets - part ii: Level set update. International Journal for Numerical Methods in Engineering, 53:2569-2586, 2002. 
[14] T. Belytschko, H. Chen, J. Xu, and G. Zi. Dynamic crack propagation based on loss of hyperbolicity and a new discontinuous enrichment. International Journal for Numerical Methods in Engineering, 58(12):1873-1905, 2003.

[15] G. Ventura, B. Moran, and T. Belytschko. Dislocations by partition of unity. International Journal for Numerical Methods in Engineering, 62(11):1463-1487, 2005.

[16] E. Samaniego and T. Belytschko. Continuum-discontinuum modelling of shear bands. International Journal for Numerical Methods in Engineering, in Press, 2004.

[17] T. Belytschko, J. Fish, and B. Englemann. A finite element method with embedded localization zones. Computer Methods in Applied Mechanics and Engineering, 70:59-89, 1988 .

[18] F. Armero and K. Garikipati. An analysis of strong discontinuities in multiplicative finite strain plasticity and their relation with the numerical simulation of strain localization in solids. International Journal of Solids and Structures, 33(20-22):2863-2885, 1996.

[19] J. Oliver, M. Cervera, and O. Manzoli. Strong discontinuities and continuum plasticity models: the strong discontinuity approach. International Journal of Plasticity, 15:319351, 1999 .

[20] S. Li, W. K. Liu, A. J. Rosakis, T. Belytschko, and W. Hao. Mesh free galerkin simulations of dynamic shear band propagation and failure mode transition. International Journal of Solids and Structures, 39:1213-1240, 2002.

[21] S. Li, W. Hao, and W.K. Liu. Mesh-free simulations of shear banding in large deformation. International Journal of Solids and Structures, 37:7185-7206, 2000.

[22] S. Hao, W.K. Liu, and C.T. Chang. Computer implementation of damage models by finite element and meshfree methods. Computer Methods in Applied Mechanics and Engineering, 187(3-4):401-440, 2000.

[23] P. Krysl and T. Belytschko. The element free galerkin method for dynamic propagation of arbitrary 3-D cracks. International Journal for Numerical Methods in Engineering, 44 (6):767-800, 1999.

[24] D. Organ, M. Fleming, T. Terry, and T. Belytschko. Continuous meshless approximations for nonconvex bodies by diffraction and transparency. Computational Mechanics, 18:225235, 1996.

[25] T. Rabczuk and T. Belytschko. Cracking particles: A simplified meshfree method for arbitrary evolving cracks. International Journal for Numerical Methods in Engineering, 61(13):2316-2343, 2004.

[26] T. Rabczuk and T. Belytschko. A three dimensional large deformation meshfree method for arbitrary evolving cracks. Computer Methods in Applied Mechanics and Engineering, in progress. 
[27] T. Belytschko, Y. Guo, W.K. Liu, and S.P. Xiao. A unified stability analysis of meshfree particle methods. International Journal for Numerical Methods in Engineering, 48:1359$1400,2000$.

[28] T. Rabczuk, T. Belytschko, and S.P. Xiao. Stable particle methods based on lagrangian kernels. Computer Methods in Applied Mechanics and Engineering, 193:1035-1063, 2004.

[29] T. Belytschko, Y.Y. Lu, and L. Gu. Element-free galerkin methods. International Journal for Numerical Methods in Engineering, 37:229-256, 1994.

[30] W.M. Wang, L.J. SLuys, and R. deBorst. Viscoplasticity for instabilities due to strain softening and strain-rate softening. International Journal for Numerical Methods in Engineering, 40(20):3839-3864, 1997.

[31] R.J. Clifton, J. Duffy, and K.A. Hartley. On critical conditions for shear band formation at high strain rates. Scripta Metallugrica, 18(5):443-448, 1984.

[32] T.W. Wright and J.W. Walter. The asymptotic structure of an adiabatic shear band in antiplane motion. Journal of the Mechanics and Physics of Solids, 44(1):77-97, 1996.

[33] T. Belytschko, W. K. Liu, and B. Moran. Nonlinear Finite Elements for Continua and Structures. John Wiley and Sons, Chichester, 2000.

[34] M. Silhavy. The Mechanics and Thermodinamics of continuous media. Springer Verlag, Berlin, 1997.

[35] D.E. Grady. Dissipation in adiabatic shear bands. Mechanics of Materials, 17:289-293, 1994.

[36] K. Minnaar and M. Zhou. An analysis of the dynamic failure resistance of structural metals. Journal Mechanics and Physics of Solids, 46(10):2155, 21701998.

[37] S. Nemat-Nasser. Plasticity. Cambridge, University Press, Cambridge, UK, 2004.

[38] J. Dolbow and T. Belytschko. Volumetric locking in the element free galerkin method. International Journal for Numerical Methods in Engineering, 46:925-942, 1999.

[39] J.S. Chen, S. Yoon, H. Wang, and W.K. Liu. An improved reproducing kernel method for nearly incompressible finite elasticity. Computer Methods in Applied Mechanics and Engineering, 181(1-3):117-145, 2000.

[40] T. J. R. Hughes. Generalization of selective integration procedures to anisotropic and nonlinear media. International Journal for Numerical Methods in Engineering, 15(9): $1413-1418,1980$.

[41] R.J. Flory. Thermodynamic relations for highly elastic materials. Transactions of the Faraday Society, 57:829-838, 1961.

[42] A.N. Brooks and T.J.R. Hughes. Streamline upwind/petrov-galerkin formulations for convective dominated flows with particular emphasis on the incompressible navier-stokes equations. Computer Methods in Applied Mechanics and Engineering, 32:199-259, 1982. 
[43] T. J. R. Hughes, L.P. Franca, and G.M. Hulber. A new finite element formulation for computational fluid-dynamics: Viii the galerkin/least squares method for advective diffusive equations. Computer Methods in Applied Mechanics and Engineering, 73:173189,1989

[44] J. F. Kalthoff and S. Winkler. Failure mode transition at high rates of shear loading. International Conference on Impact Loading and Dynamic Behavior of Materials, 1:185195, 1987.

[45] J.F. Kalthoff. Modes of dynamic shear failure in solids. International Journal of Fracture, 101:1-31, 2000.

[46] M. Zhou, G. Ravichandran, and A. Rosakis. Dynamically propagating shear bands in impact-loaded prenotched plates-i. Journal Mechanics Physics and Solids, 44:981-1006, 1996.

[47] K. Ravi-Chandar, J. Lu, B. Yang, and Z. Zhu. Failure modes transitions in polymers under high strain rate loading. International Journal of Fracture, 101:33-72, 2000.

[48] R.C. Batra and R.R. Gummalla. Effect on material and geometric parameters on deformations near the notch-tip of a dynamically loaded prenotched plate. International Journal of fracture, 101:99-140, 2000.

[49] M. Zhou, G. Ravichandran, and A. Rosakis. Dynamically propagating shear bands in impact-loaded prenotched plates-ii. Journal Mechanics Physics and Solids, 44:1007-1032, 1996.

[50] R.C. Batra and M.V.S. Ravisankar. Three-dimensional numerical simulation of the kalthoff experiment. International Journal of fracture, 105:161-186, 2000.

[51] G.R. Johnson and W.H. Cook. A constitutive model and data for metals subjected to large strains, high strain rates, and high temperatures. In Proc. 7th International Symp. on Ballistics, 1983.

[52] P. Diez, M. Arroyo, and A. Huerta. Adaptivity based on error estimation for viscoplastic softening materials. Mechanics of Cohesiv-Frictional Materials, 5:87-112, 2000.

[53] T. Rabczuk and T. Belytschko. Adaptivity for structured meshfree particle methods in 2D and 3D. International Journal for Numerical Methods in Engineering, 63(11):1559-1582, 2005 .

[54] P.M.A. Areias. Finite element technology, damage modeling, contact constraints and fracture analysis. Doutoramento, FEUP - Faculdade de Engenharia da Universidade do Porto, Rua Dr. Roberto Frias s/n 4200-465 Porto, Portugal, 2003. www.fe.up.pt.

[55] C. Miehe and M. Lambrecht. A two-scale finite element relaxation analysis of shear bands in non-convex inelastic solids: small-strain theory for standard dissipative materials. Computer Methods in Applied Mechanics and Engineering, 192:473-508, 2003.

[56] V.F. Nesterenko, M.A. Meyers, and T.W. Wright. Self-organization in the initiation of adiabatic shear bands. Acta mater., 1998. 
[57] Q. Xue, V.F. Nesterenko, and M.A. Meyers. Evaluation of the collapsing thick-walled cylinder technique for shear-band spacing. International Journal of Impact Engineering, 2003.

[58] M.A. Meyers, V.F. Nesterenko, J.C. LaSalvia, and Q. Xue. Shear localization in dynamic deformation of materials: microstructural evolution and self-organization. Material Science and Engineering, 2001.

[59] C.J. Shih, M.A. Meyers, and V.F. Nesterenko. High-strain-rate deformation of granular silicon carbide. Acta mater., 1998.

[60] T. Belytschko, N. Moes, S. Usui, and C. Parimi. Arbitrary discontinuities in finite elements. International Journal for Numerical Methods in Engineering, 50(4):993-1013, 2001. 